\title{
COLLECTIVE RISK-TAKING DeCISIONS WITH HETEROGENEOUS BELIEFS
}

\author{
CHRISTIAN GOLLIER
}

CESIFo Working PAPER No. 909

CATEGORY 8: RESOURCES AND ENVIRONMENT

APRIL 2003

\footnotetext{
An electronic version of the paper may be downloaded

- from the SSRN website: $\quad$ www.SSRN.com

- from the CESifo website: www.CESifo.de
} 


\title{
COLLECTIVE RISK-TAKING DECISIONS WITH HETEROGENEOUS BELIEFS
}

\begin{abstract}
Suppose that a group of agents having divergent expectations can share risks efficiently. We examine how this group should behave collectively to manage these risks. We show that the beliefs of the representative agent is in general a function of the group.s wealth level, or equivalently, that the representative agent has a state-dependent utility function. We define the individual degree of pessimism as an index measuring probability differences across states. We show that the degree of pessimism of the representative agent is the mean of the individual ones weighted by their index of absolute risk tolerance. From this central result, we show how increasing disagreement on the state probability affects the state probability of the representative agent. We show that the divergence of opinions about the probability of occurence of a boom may help solving the equity premium puzzle.
\end{abstract}

JEL Code: D7, D81.

Keywords: aggregation of beliefs, state-dependent utility, efficient risk sharing, pessimism, disagreement, asset pricing, portfolio choices.

\author{
Christian Gollier \\ University of Toulouse \\ IDEI \\ Place Anatole France \\ 31042 Toulouse Cedex \\ France \\ gollier@cict.fr
}

Preliminary version. I thank Jean-Marc Tallon for helpful discussions. 


\section{Introduction}

People have divergent opinions on a wide range of subjects, from the outcome of an election or of a war, the profitability of a new technology to the risk of global warming. Suppose that this heterogeneity of beliefs does not come from asymmetric information but rather from intrinsic differences in how to view the world. People agree to disagree, which implies that prices and observed behaviors of other market participants do not generate any Bayesian updating of individual beliefs. We examine how the group as a whole will behave towards risk. Aggregating beliefs when agents differ on their expectations is useful to solve various economic questions, from asset pricing to cost-benefit analyses of collective risk prevention.

The attitude towards risk of a group of agents depends upon how risk is allocated in the group. For example, if an agent is fully insured by other agents, it is intuitive that this agent's beliefs should not affect the social welfare function. Only those who bear a share of the risk should see their expectations be taken into account on the collective risk decision. In this paper, we assume that risks can be allocated in a Pareto-efficient way in the group. In such a situation, the willingness to take risk is increasing in the Arrow-Pratt index of absolute risk tolerance. It implies that the beliefs of agents with a larger risk tolerance have a larger impact on how individual expectations are aggregated. At the limit, those with a zero risk tolerance do not influence the group's expectations.

The properties of the socially efficient probability distribution are derived from the characteristics of the efficient allocation of risk in the group, such as the one derived from the competitive allocation with complete Arrow-Debreu markets. Borch (1960) and Wilson (1968) were the first to characterize the properties of Pareto-efficient risk sharings when agents have identical beliefs. ${ }^{1}$ Leland (1980) examined the competitive equilibrium asset portfolios when agents have different priors on the distribution of state probabilities. If $\pi(s)$ and $p(s, \theta)$ denote respectively the price of the Arrow-Debreu security associated to state $s$ and the subjective probability associated to that state by agent $\theta$, the demand for that security depends negatively upon $\pi(s) / p(s, \theta)$,

Hara and Kuzmics (2001) provide new results about how to aggregate risk aversion when beliefs are homogeneous. 
the state price per unit of subjective probability. Ex ante, the degree of riskiness of the optimal portfolio for agent $\theta$ depends upon the volatility of this true price with respect to $s$. For example, if $\pi($.$) and p(., \theta)$ coincide, that is if agent $\theta$ perceives all asset prices as actuarially priced, then he should buy a risk free portfolio. More generally, the riskiness of the optimal portfolio of agent $\theta$ is increasing in the sensitivity of $\pi(s) / p(s, \theta)$ to changes in $s$. It can be measured locally by the difference between $\delta^{\pi}(s)=-\pi^{\prime}(s) / \pi(s)$ and $\delta^{p}(s, \theta)=-p_{s}(s, \theta) / p(s, \theta)$. This difference is a measure of the degree of divergence between the market beliefs and the beliefs of agent $\theta$. If larger states are better, $\delta^{\pi}$ and $\delta^{p}$ measure respectively the market and the individual degrees of pessimism. If the agent is more pessimist than the market, his demand for state-contingent consumption will be decreasing with $s$. Seen from ex ante, this means that the agent bears a risk. It is increasing in his degree of pessimism. We conclude that information about individual degrees of pessimism are more useful than the individual probability distributions to determine efficient allocations of risk in the economy. From this observation, we concentrate much of our attention in this paper to the effect of the heterogeneity of the individual degrees of pessimism on the socially efficient group's degree of pessimism.

We show that the beliefs of the representative agent in this economy is the mean of the individual degrees of pessimism weighted by their absolute risk tolerance. This central property of aggregation has various consequences. For example, the representative agent usually has a state-dependent utility function, despite the fact that all members of the group have state-independent preferences. The representative agent has state-additive preferences, but the terms of the sum cannot be written as a product of a probability that would depend only upon the state by a utility that would depend only upon consumption. Drèze (2001) and Drèze and Rustichini (2001) examine the effect of the state dependency of the utility function for risk management and risk transfers. Another way to interpret this result is that the collective probability distribution depends upon the aggregate wealth level.

Our aggregation result also implies that the collective pessimism is within the range of the individual degrees of pessimism. This result must be compared to the observation that the state probability used by the representative agent does not need to be in between the smallest and the largest state probabilities of the agents. Another consequence of this result is that, the dispersion of individual subjective probabilities will affect the collective degree of pessimism. More dispersed individual (Pareto-weighted) subjective 
probabilities imply more dispersed individual state consumption levels. If absolute risk tolerance is increasing, this will bias the collective degree of pessimism towards the degree of pessimism of those who have a large subjective probability for that state. Thus, decreasing absolute risk aversion is the condition that determines the effect of locally increasing disagreement when the mean degree of pessimism remains constant, at least locally.

We also compare the degree of pessimism of the representative agent with the degree of pessimism that would be derived from the Pareto-weighted mean of individual subjective probabilities. We show that increased disagreement makes the degree of pessimism of the representative agent larger if the derivative of absolute risk tolerance is smaller than unity, a plausible assumption on preferences. This result is more general than the one obtained by Varian (1985) and Ingersoll (1987) who compared two states of nature with the same mean Pareto-weighted individual subjective probabilities.

All these results describe how the heterogeneity of beliefs affects the local slope of the collective distribution function. Going from the local analysis to a global one, it is necessary to describe the structure of disagreements across states. This would be useful to determine whether the collective distribution function be stochastically dominated by the mean subjective distribution. Cecchetti, Lam and Mark (2000) and Abel (2002) examine the effect of a change in the beliefs of the representative agent on the equity premium. Contrary to us, they assume that all agents have the same beliefs that deviate from what could be inferred from the existing data. However, their work is useful to us because once the beliefs of the representative agent is obtained, our model becomes equivalent to an economy with homogeneous beliefs. It is not true in general that a first-order-stochastically dominated shift in the subjective distribution of aggregate consumption raises the equity premium. Abel (2002) defines the notion of uniform pessimism by a leftward translation of the objective distribution of the aggregate consumption. He shows that uniform pessimism raises the equity premium. We provide another result which states that transferring probability mass from the wealthiest states uniformly to the other states also unambiguously raises the equity premium. It happens that the heterogeneity of beliefs where most of the disagreement is about the probability of the wealthiest states generates precisely this change in the subjective distribution of the representative agent. We provide a numerical illustration that shows that a disagreement on the likelihood of a boom may have a sizeable positive effect on the equity premium. In a plausible simulation, it multiplies the equity premium by 4 . The bad news is that 
the equity premium is reduced when most of the disagreement is about the likelihood of a krach. Calvet, Grandmont and Lemaire (2001) also examine the effect of heterogenous beliefs on the equity prmium. They are able to sign this effect when the relative risk aversion of the representative agent is decreasing with average wealth.

The structure of the paper is as follows. In section 2, we characterize the optimal structure of the portfolio in an Arrow-Debreu economy. We define our concept of pessimism. Section 3 is devoted to the description of the aggregation problem when agents have heterogeneous preferences and beliefs. We show how to aggregate individual risk tolerances in this framework in section 4 , whereas section 5 is devoted to the aggregation rule for individual beliefs. In section 6 , we examine the problem of the multiplicative separability between utility and probability. In section 7 , we define our concept of increasing disagreement, and we determine its effect on the collective degree of pessimism. Section 8 provides a global analysis of the effect of the heterogeneity of beliefs on the collective probability distribution and the equity premium. Finally, we present concluding concluding remarks in section 9 .

\section{Properties of optimal individual portfolios}

In this section, we consider an agent $\theta$ who extracts utility from consuming a single consumption good. The model is static with one decision date and one consumption date. At the decision date, there is some uncertainty about the state of nature $s$ that will prevail at the consumption date. The set of possible states of nature is denoted $S \subset R$. The agent is an expected-utility maximizer with a state-dependent utility function $u(., ., \theta): R \times S \rightarrow R$ where $u(c, s, \theta)$ is the utility of agent $\theta$ consuming $c$ in state $s$. We assume that $u_{c}=\partial u / \partial c$ is continuously differentiable in $(c, s)$, and is nonincreasing in $c$ to express risk aversion. We make no specific assumption on the state dependency of the marginal utility of consumption. We also assume that agent $\theta$ has beliefs that can be represented by a density function $p(., \theta): S \rightarrow R^{+}$which is differentiable with respect to $s$. This subjective probability density is not unique, for it is always possible to write $\mathfrak{b}(s, \theta) \mathfrak{b}\left(c_{\mathbf{R}^{s}}, \theta\right)=p(s, \theta) u(c, s, \theta)$ where functions $\boldsymbol{\emptyset}$ and $\boldsymbol{b}$ are arbitrarily chosen with ${ }_{S} \boldsymbol{b}(s, \theta) d s=1$.

There is a complete set of Arrow-Debreu securities in the economy. The equilibrium price of the Arrow-Debreu security associated to state $s$ is denoted $\pi(s, \theta)>0$. It means that agent $\theta$ must pay $\pi(s, \theta)$ ex-ante to receive 
one unit of the consumption good if and only if state $s$ occurs. ${ }^{2}$ We normalize prices in such a way that ${ }_{S} \pi(s, \theta)=1$. The agent is endowed with wealth $z$ ex ante to finance future consumption. We examine the optimal portfolio of Arrow-Debreu securities for agent $\theta$. It is the solution of the following program:

$$
\begin{gathered}
V(z, \theta)=\max _{C_{(.)}}{ }_{S} p(s, \theta) u(C(s), s, \theta) d s \\
\text { s.t. } \quad{ }_{S} \pi(s, \theta) C(s) d s=z .
\end{gathered}
$$

The optimal portfolio is characterized by function $c$, where $C^{*}(s)=c(z, s, \theta)$ is the demand of agent $\theta$ endowed with initial wealth $z$ for the Arrow-Debreu security associated to state $s$. The value function $V$ is such that $V(z, \theta)$ is the maximum expected utility that agent $\theta$ can extract from initial wealth $z$. We suppose that a solution to this program exists. Given the concavity of $u$ with respect to $c$, it is unique.

The following first-order condition is necessary and sufficient: for all $s \in$ $S$, for all $z$,

$$
\ln p(s, \theta)+\ln u_{c}(c(z, s, \theta), s, \theta)=\ln \pi(s, \theta)+\ln V_{z}(z, s) .
$$

Fully differentiating this condition with respect to $z$ and using the budget constraint (2) yields that

$$
c_{z}(z, s, \theta)=\frac{\mathrm{R}}{{ }_{S} \pi(t, \theta) T^{u}(c(z, t, \theta), t, \theta) d t},
$$

where $T^{u}$ is the degree of absolute risk tolerance measured by

$$
T^{u}(c, s, \theta)=-\frac{\partial}{\partial c} \ln u_{c}(c, s, \theta)^{\lrcorner-1}=-\frac{u_{c}(c, s, \theta)}{u_{c c}(c, s, \theta)} .
$$

An increase in initial wealth raises the demand for Arrow-Debreu securities which are thus normal goods. The increase in demand is proportional to the degree of absolute risk tolerance measured in the corresponding state.

\footnotetext{
${ }^{2}$ The dependency of state prices to the type $\theta$ of the agent is made for the sake of generality and is not essential in this paper. It allows for exploring the sensitivity of the optimal portfolio to changes in the distribution of contingent prices.
} 
One can define the degree of risk tolerance on wealth $T^{V}$ as

$$
T^{V}(z, \theta)=-\frac{\partial}{\partial z} \ln V_{z}(z, \theta)^{\lrcorner-1}=-\frac{V_{z}(z, \theta)}{V_{z z}(z, \theta)} .
$$

This would be useful for example to determine the optimal risk to be taken prior to the decision period that is considered in this section. Using the first-order condition (3) and property (4) yields the following property:

Z

$$
T^{V}(z, \theta)={ }_{S} \pi(s, \theta) T^{u}(c(z, s, \theta), s, \theta) d s .
$$

The absolute risk tolerance on wealth is the "risk-neutral" mean of the statedependent absolute risk tolerances on consumption. This property has several implications for dynamic portfolio management that are examined in Gollier (2001, chapter 14). For example, $V$ inherits the property of increasing absolute tolerance from the state-dependent utility functions $u(., s, \theta)$.

The riskiness of the optimal portfolio can be evaluated by the variability of consumption across states. A local measure of such variability is given by $c_{s}(z, s, \theta)$. Fully differentiating the first-order condition (3) with respect to $s$ and using again the budget constraint (2) provides a characterization of the optimal local risk exposure:

$$
c_{s}(z, s, \theta)=T^{u}(c(z, s, \theta), s, \theta)\left[{ }^{s} \delta^{\pi}(s, \theta)-{ }^{s} \delta^{p}(s, \theta)-{ }^{s} \delta^{u}(c(z, s, \theta), s, \theta)\right],
$$

with

$$
\begin{aligned}
& { }^{s} \delta^{\pi}(s, \theta)=-\frac{\partial}{\partial s} \ln \pi(s, \theta), \\
& { }^{s} \delta^{p}(s, \theta)=-\frac{\partial}{\partial s} \ln p(s, \theta),
\end{aligned}
$$

and

$$
{ }^{s} \delta^{u}(c, s, \theta)=-\frac{\partial}{\partial s} \ln u_{c}(c, s, \theta) .
$$

The deltas measure the rate at which functions $\pi, p$, and $u_{c}$ decrease when considering increasing states $s$. The simplest case is when the utility function is state independent $\left({ }^{s} \delta^{u} \equiv 0\right)$ and when state prices are proportional to state probabilities $\left({ }^{s} \delta^{\pi} \equiv{ }^{s} \delta^{p}\right)$. In this special case, property (7) just means that the risk-averse investor purchases a risk free portfolio $\left(c_{s} \equiv 0\right)$. If we relax the assumption that asset prices are actuarial, property (7) implies that 
consumption is increasing with the state if states corresponding to a larger $s$ have a smaller price per unit of probability: $c_{s}>0$ if $\pi / p$ is decreasing in $s$, or if ${ }^{s} \delta^{\pi}$ is larger than ${ }^{s} \delta^{p}$.

It is good to give names to these concepts. To do this, let us rank states in such a way that larger states correspond to larger ex post utility for agent $\theta$. Larger states are better states from the point of view of agent $\theta$. Under this assumption, ${ }^{s} \delta^{p}(s, \theta)$, which measures the rate at which subjective state probabilities for agent $\theta$ decrease as $s$ improves, is a local measure of pessimism. Similarly, ${ }^{s} \delta^{\pi}(s, \theta)$ is a measure of the market's degree of pessimism implicit in the pricing kernel. Thus, under the assumption that $\delta^{u} \equiv 0$, property (7) implies that consumption is increasing with the state if the investor is less pessimistic than the market about the likelihood of high/good states. Ex ante, this means that agent $\theta$ takes risk. As seen in (7), the optimal local risk exposure is proportional to the agent's local degree of absolute risk tolerance $T^{u}$.

When ${ }^{s} \delta^{u}$ is positive, that is, when the marginal utility of consumption is decreasing with the state, the willingness to consume more in higher state is reduced, as seen in the right-hand side of equation (7). This reinforces the effect of pessimism. Equation (7) tells us that what matters to determine the optimal riskiness of the portfolio is the sum of ${ }^{s} \delta^{p}$ and ${ }^{s} \delta^{u}$. In other words, observing household portfolios does not allow for disentangling household pessimism from the state-dependency of utility. This is inherent to the definition of state-dependent expected utility, where it is hard to make the difference between probabilities and utils. ${ }^{3}$ Thus, ${ }^{s} \delta^{p}+{ }^{s} \delta^{u}=-\frac{\partial}{\partial s} \ln p u_{c}$ will be called the corrected degree of pessimism.

We can also easily examine the sensitivity of the optimal portfolio $c$ and of the marginal value of wealth $V_{z}$ to changes of the model specification. One can examine the effect of a change in the beliefs $p$ of the investor, of a change in the investor's degree of risk tolerance, or of a change in asset prices. Fully differentiating the first-order condition (3) with respect to $\theta$ and using the budget constraint (2) yields

$c_{\theta}(z, s, \theta)=T^{u}(c(z, s, \theta), s, \theta){ }^{\mathbf{f}_{\theta}} \delta^{V}(z, \theta)+{ }^{\theta} \delta^{\pi}(s, \theta)-{ }^{\theta} \delta^{p}(s, \theta)-{ }^{\theta} \delta^{u}(c(z, s, \theta), s, \theta)^{\mathfrak{\alpha}}$

\footnotetext{
${ }^{3}$ See Drèze and Rustichini (2001) for a discussion on this point.
} 
and

$$
\begin{aligned}
& { }^{\theta} \delta^{V}(z, \theta)={ }_{\text {def }} \quad \overline{\mathrm{R}} \frac{\partial}{\partial \theta} \ln V_{z}(z, \theta) \\
& =\frac{{ }_{\pi(z, s)\left[T^{u}(c, s, \theta)\left({ }^{\theta} \delta^{p}\left(s_{\mathrm{R}} \theta\right)+{ }^{\theta} \delta^{u}(c, s, \theta)\right)+{ }^{\theta} \delta^{\pi}(s, \theta)\left(c-T^{u}(c, s, \theta)\right)\right] d s}^{\pi(z, s) T^{u}(c, s, \theta) d s}}{\text {, }}
\end{aligned}
$$

where $c$ is evaluated at $(z, s, \theta)$ and

$$
\begin{aligned}
& { }^{\theta} \delta^{\pi}(s, \theta)=-\frac{\partial}{\partial \theta} \ln \pi(s, \theta) \\
& { }^{\theta} \delta^{p}(s, \theta)=-\frac{\partial}{\partial \theta} \ln p(s, \theta),
\end{aligned}
$$

and

$$
{ }^{\theta} \delta^{u}(c, s, \theta)=-\frac{\partial}{\partial \theta} \ln u_{c}(c, s, \theta) .
$$

The mu functions measure the degree of heterogeneity in the model specifications, respectively for $\pi, p$, and $u_{c}$. Equation (11) shows how the optimal portfolio $c$ is affected by a change in asset prices $\left({ }^{\theta} \delta^{\pi}\right)$, a change in beliefs $\left({ }^{\theta} \delta^{p}\right)$, or by a change in the risk preferences $\left({ }^{\theta} \delta^{u}\right)$. In equation (12), we characterize their effect on the marginal value of wealth $\left({ }^{\theta} \delta^{V}\right)$. These equations will be central later in this paper. Some comments are in order here. First, observe from (11) that the sensitivity of the optimal portfolio to changes in specification is proportional to the investor's degree of absolute risk tolerance. In a sense, risk aversion is an index of stubbornness. Second, consider the effect of a change in beliefs $\left({ }^{\theta} \delta^{\pi}={ }^{\theta} \delta^{u} \equiv 0\right)$. Observe that in this case ${ }^{\theta} \delta^{V}$ is a weighted sum of the rate of reduction in state probabilities ${ }^{\theta} \delta^{p}$, which can be positive or negative. Suppose that ${ }^{\theta} \delta^{V}$ is positive. Then, there must exist some states for which ${ }^{\theta} \delta^{p}>0$ and ${ }^{\theta} \delta^{V}-{ }^{\theta} \delta^{p}>0$. It implies from (11) that the investor would increase his demand for consumption in these states in spite of the fact that he believes that they are less likely to occur! This observation provides an important message for the remaining of this paper: what matters to determine the optimal exposure to risk are not the state probabilities per se. The important concept is the investor's degree of pessimism relative to the market's one. ${ }^{4}$

\footnotetext{
${ }^{4}$ Since the seminal work by Rothschild and Stiglitz (1971), there has been an important litterature on the effect of a change in risk on the optimal demand for risky assets. Gollier (1995) derives the necessary and sufficient condition for a change in risk to raise the demand for the risky asset by all risk-averse agents.
} 
It is noteworthy that we did not use assumption ${ }^{\mathrm{R}} p(s, \theta) d s=1$ to derive the results presented in this section. This remark will be important in the remaining of this paper. Indeed, we will use alternative interpretations of program (1) where the $p$ function does not sum up to unity.

In the next section, we reconsider the same portfolio problem, but we go from the individual level to the collective one. Following Constantinides (1982), this raises the question problem of how to aggregate individual preferences and beliefs.

\section{The aggregation problem}

We consider an economy or a group of heterogeneous agents indexed by $\theta$ in a type set $\Theta$. Types are distributed according to cumulative distribution function $H: \Theta \rightarrow[0,1]$. Agents are heterogeneous in terms of risk attitude, beliefs and wealth. This is reflected in the dependency of the $u, p$ and $\omega$ functions to $\theta$. $E$ denotes th $\mathrm{R}$ expectation operator with respect to $\theta$ with distribution $H$, i.e., $E f(\boldsymbol{\theta})={ }_{\Theta} f(\theta) d H(\theta)$ is the mean of $f(\boldsymbol{\theta})$ with respect to the type distribution $H$.

The group faces a portfolio choice that is equivalent to the one described in the previous section. A consumption plan is described by a function $C(.,):. S \times \Theta \rightarrow R$. The consumption per capita in state $s$ is denoted $z(s)$ :

$$
E C(s, \vartheta)=z(s)
$$

for all $s \in S$.

The crucial assumption of this paper is that the group can allocate risks efficiently among its members. An allocation $C(.,):. S \times \Theta \rightarrow R^{+}$is Paretoefficient if it is feasible and there is no other feasible allocation that raises the expected utility of at least one member without reducing the expected utility of the others. For a given Pareto-weight function $\lambda():. \Theta \rightarrow R^{+}$, normalized in such a way that $E \lambda(\theta)=1$, the group would select the portfolio of Arrow-Debreu securities and the allocation of the risk within the group that maximize the weighted sum of the members' expected utility under the feasibility constraint:

$$
\max _{C} E^{\cdot} \lambda(\boldsymbol{Z}){ }_{S}^{\mathbf{Z}} p(s, \boldsymbol{\vartheta}) u(C(s, \boldsymbol{\vartheta}), s, \boldsymbol{\vartheta}) d s^{\lrcorner}
$$




$$
\text { s.t. }{ }_{S} \pi(s, \boldsymbol{Z}) E C(s, \boldsymbol{\mathrm { h }})-\omega(s, \boldsymbol{\vartheta})^{\mathrm{i}} d s=0 .
$$

It is useful to decompose this decision problem into two stages. Define

$$
v(z, s)=\max _{c^{0}(z, ., \theta)} E^{\mathrm{h}} \lambda(\vartheta) p(s, \vartheta) u(c(z, s, \vartheta), s, \vartheta)^{\mathbf{i}} \quad \text { s.t. } E c(z, s, \boldsymbol{\theta})=z \text {. }
$$

In this cake-sharing problem, $z$ represents the consumption per capita, and $v(z, s)$ is the maximum sum of subjective-probability-weighted utility in state $s$. The second stage is a collective portfolio problem in which the group selects the state-dependent sizes of the cake that maximizes the sum of $v$ across the states:

Z

$$
\max _{z(.)} v(z(s), s) d s \quad \text { s.t. }{ }_{S} \pi(s)[z(s)-\omega(s)] d s=0,
$$

where $\omega(s)=E \omega(s, \vartheta)$ is the endowment per capita in state $s$. Obviously, combining these two-stage problems generates the solution to program (17), with $C(s, \theta)=c(z(s), s, \theta)$.

This decomposition is interesting for various reasons. Notice first that the $v$ function describes the risk attitude and beliefs of the representative agent in the sense of Constantinides (1982). It is implicitly a product of the collective state probabilities by the collective utility for consumption per capita. However, it is not true in general that the representative agent has preferences and beliefs that are multiplicatively separable as in the standard expected utility model. The implicit subjective probabilities of the representative agent may be wealth-dependent, or equivalently, the utility function of the representative agent may be state-dependent. This is why we considered the non-separable case in the previous section. Being neither a probability nor a utility, but rather the contribution of the state to ex-ante expected utility, $v$ will hereafter be referred to as the contribution function. The existence of such a contribution function for the representative agent extends previous aggregation results by Wilson (1968) and Constantinides (1982) to the case of heterogeneous preferences. Such an extension is a direct consequence of the additive separability of the individual preference functionals.

Second, observe that the portfolio stage (20) is a special case of the problem that we solved in the previous section. It implies in particular that the degree of riskiness of the optimal collective portfolio - which is measured by 
$z^{\prime}(s)$ - is a function of the collective risk tolerance ${ }^{5}$

$$
T^{v}(z, s)=-\frac{\partial}{\partial z} \ln v_{z}(z, s)^{-1}
$$

and of the collective (corrected) degree of pessimism defined as

$$
{ }^{s} \delta^{v}(z, s)=-\frac{\partial}{\partial s} \ln v_{z}(z, s)
$$

These characteristics of the preferences and beliefs of the representative agent must be derived from the definition (19) of the $v$ function. The objective of this paper is to link $T^{v}$ and $\delta^{v}$ to the distribution of individual traits $T^{u}$ and $\delta^{p}$ in the group to derive various implications from these links. This work is much simplified by the following central insight relative to program (19). This cake-sharing problem is not much different from the individual portfolio problem (1). In these two maximization programs, both the objective function and the constraint is additively separable, either with respect to states $s$ for (1), or with respect to types $\theta$ for (19). Using the paradigm of the veil of ignorance, the cake-sharing problem is in fact a portfolio problem. ${ }^{6}$ Under the veil of ignorance, the uncertainty is about how types will be allocated among the group's members. Suppose that in state $s$, agents believe that the probability to get a type within $[\theta, \theta+d \theta]$ equals $\lambda(\theta) p(s, \theta) d H(\theta) / E \lambda(\boldsymbol{\theta}) p(s, \boldsymbol{\theta})$. Then, given a specific cake per capita $z$, the ex-ante identical agents will unanimously favor the selection of the cake-sharing rule $c(z, s, \boldsymbol{\theta})$ - actually a portfolio of type-contingent claims - that maximizes their expected utility described in (19). Because this is a zero-sum game, the type-prices implicit in the budget/feasibility constraint $E \pi(\vartheta) c(z, s, \vartheta)=z$ is $\pi(\theta) \equiv 1$.

The equivalence property is central because it allows us to use all properties that we derived earlier. This simple exercise consisting in reinterpreting program (1) as a cake-sharing problem requires some words of caution, however. In the state-contingent cake-sharing problem, the role of $\theta$ and $s$ are reversed compared to the portfolio problem. It is now $\theta$ which is random, and $s$ becomes a parameter. The comparative statics exercise of a change in the parameter $\theta$ on the optimal portfolio that we performed at the end of section

\footnotetext{
${ }^{5} \mathrm{Nau}$ (2002) discusses the measure of risk aversion in the small and in the large in the case of state-dependent utilities.

${ }^{6}$ Gollier (2001) summarizes the links between the two decision problems when agents share common beliefs.
} 
2 is now useful to determine how the efficient cake-sharing is affected by the choice of $s$. This will be useful in a second step to determine how the collective subjective probabilities change across states $\left({ }^{s} \delta^{v}\right)$. The interpretation of $z$ is also transformed. In the portfolio problem, $z$ is the ex-ante wealth of the investor, whereas in the cake-sharing problem, $z(s)$ is the wealth per capita available in state $s$ ex post. Finally, the $V$ function in the portfolio problem (1) corresponds to the contribution function $v$ in the cake-sharing problem (19).

\section{Aggregation of risk aversion}

As a first illustration of the usefulness of the equivalence of the portfolio and cake-sharing problems, let us consider the problem of determining the properties of the efficient sharing of the risk in the group. It is characterized by $\partial c / \partial z$ which determines how differences of wealth per capita across states must be allocated to differences in individual consumption levels. Using condition (4) by equivalence, it is equal to

$$
\frac{\partial c}{\partial z}(z, s, \theta)=\frac{T^{u}(c(z, s, \theta), s, \theta)}{E T^{u}(c(z, s, \vartheta), s, \vartheta)} .
$$

One can interpret this property of the efficient risk-sharing rule as follows: suppose that there are two states of nature that are perceived to be identical by all agents, expect for the mean income $z$. In particular, probabilities and conditional utility functions are identical for the two states. Equation (23) shows how to allocate the collective wealth differential in the two states. Observe that the positiveness of the right-hand side of (23) means that individual consumption levels are all procyclical. Adapting Wilson (1968) to the case of state-dependent preferences, more risk-tolerant agents should bear a larger fraction of the collective risk. More precisely, for any $s \in S$, their optimal consumption plan will single-cross from below the optimal consumption plan of any less risk-tolerant agents in the $(z, c)$ diagram: $\forall\left(\theta, \theta^{\prime}\right) \in \Theta^{2}: T(c, s, \theta) \geq T\left(c, s, \theta^{\prime}\right)$ for all $c$ implies that

$$
c(z, s, \theta)=c(z, s, \theta) \Longrightarrow \frac{\partial c}{\partial z}(z, s, \theta) \geq \frac{\partial c}{\partial z}\left(z, s, \theta^{\prime}\right) .
$$

This property can be better understood if first we recall the mutuality principle. In the classical case with state-independent utility functions and homogeneous beliefs, it states that efficient individual consumption levels depend 
upon the state only through the wealth per capita $z$. Its economic interpretation is that all diversifiable risks are eliminated through sharing. In this classical case, the wealth level per capita $z$ is a sufficient statistic for efficient individual consumption levels, and property (24) shows that agent $\theta$ bears a larger share of the aggregate risk than agent $\theta^{\prime}$. He consumes relatively more in the wealthier states, and relatively less in the poorer ones.

From this efficient collective risk-sharing rule, it is easy to derive the degree of risk tolerance of the group as a whole. In parallel to equation (6), it is written as

$$
T^{v}(z, s)=E T^{u}(c(z, s, \vartheta), s, \vartheta) .
$$

The group's absolute risk tolerance is the mean of its members' tolerance. We conclude that this rule already valid in the simpler Wilson's model is robust to the introduction of either heterogeneous expectations or state-contingent preferences.

\section{The group's index of pessimism}

When beliefs are homogenous and individual utility functions are stateindependent, the mutuality principle states that efficient individual consumption levels depend upon the state only through the mean wealth $z$ available in the corresponding state: $\partial c / \partial s \equiv 0$. With heterogeneous beliefs or statedependent utility functions, this result is of course not true. For example, agents will find mutually advantageous exchanges of zero-sum lotteries in order to gamble on states that they believe to be more likely than their counterpart. In the following, we examine the partial derivative of individual consumption levels with respect to states. It characterizes the efficient allocation of diversifiable risks in the group.

The efficient allocation of the diversifiable risk depends upon how the corrected degrees of pessimism differ across the members of the group. The corrected degree of pessimism of agent $\theta$ in state $(z, s)$ is defined as

$$
{ }^{s} \delta^{p u}(z, s, \theta)={ }^{s} \delta^{p}(s, \theta)+{ }^{s} \delta^{u}(c(z, s, \theta), s, \theta)
$$

The following Proposition is a direct consequence of the equivalence property together with equations (11) and (12). 
Proposition 1 The index of pessimism of the representative agent defined by (22) is a weighted mean of individual members' corrected indexes of pessimism:

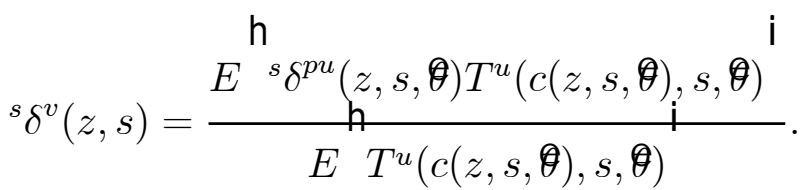

The efficient allocation of diversifiable risks satisfies the following condition:

$$
\frac{\partial c}{\partial s}(z, s, \theta)=T^{u}(c(z, s, \theta), s, \theta)\left[{ }^{s} \delta^{v}(z, s)-{ }^{s} \delta^{p u}(z, s, \theta)\right],
$$

Let us first focus on property (28). This result implies that more (corrected) pessimistic people should substitute consumption in high states for consumption in lower states. The following Proposition describes this intuitive property of efficient risk allocations. This single-crossing property in the $(s, c)$ diagram is closely related to the single-crossing property in the $(z, c)$ diagram that we described in the previous section. ${ }^{7}$

Proposition 2 Suppose that agents have the same state-independent risk tolerance : $\partial T^{u}(c, s, \theta) / \partial \theta=\partial T^{u}(c, s, \theta) / \partial s \equiv 0$. Suppose also that conditional on wealth per capita $z$, index $\theta$ ranks agents according to their degree of pessimism : $\forall\left(\theta, \theta^{\prime}\right) \in \Theta^{2}: \theta>\theta^{\prime} \Longrightarrow{ }^{s} \delta^{p}(z, s, \theta)>{ }^{s} \delta^{p}\left(z, s, \theta^{\prime}\right) \quad \forall s$. Then, all efficient allocations of diversifiable risks satisfy the single crossing property:

$$
c(z, s, \theta)=c\left(z, s, \theta^{\prime}\right) \Longrightarrow \frac{\partial c}{\partial s}(z, s, \theta) \leq \frac{\partial c}{\partial s}\left(z, s, \theta^{\prime}\right)
$$

Proof: This is a direct consequence of equation $(28)$ with ${ }^{s} \delta^{p u} \equiv{ }^{s} \delta^{p}$.

The assumption that ${ }^{s} \delta^{p}$ is uniformly increasing in $\theta$ just means that the likelihood ratio $p(s, \theta) / p\left(s, \theta^{\prime}\right)$ is decreasing in $s$ when $\theta>\theta^{\prime}$. This corresponds to the Monotone Likelihood Ration (MLR) order which is a special case of first-order stochastic dominance. When agent $\theta$ has expectations that are MLR dominated by agent $\theta^{\prime}$ 's expectations, the first will consume relatively more in low states, and relatively less in high states.

\footnotetext{
${ }^{7}$ The duality between the notion of more risk aversion and more pessimism remind us some works by Athey (2001) and Gollier (2001, chapter 7) who examine dual relationship between the concepts of "more risk aversion", the monotone likelihood ratio order, and logsupermodular functions.
} 
Condition (27) provides a nice characterization of the aggregation of individual beliefs in groups that can share risk efficiently. The group's corrected degree of pessimism can be rewritten as

$$
{ }^{s} \delta^{v}(z, s)=\mathfrak{\bigotimes}_{z, s}{ }^{s} \delta^{p u}(z, s, \vartheta),
$$

where $\unrhd_{z, s}$ is a "risk-neutral" expectation operator defined as

$$
\varliminf_{z, s} f(\boldsymbol{\theta})=\frac{\mathrm{R}_{\mathrm{R}}(\theta) T^{u}(c(z, s, \theta), s, \theta) d H(\theta)}{T^{u}(c(z, s, \theta), s, \theta) d H(\theta)} .
$$

For each state, the (corrected) index of pessimism of the representative agent is a weighted mean of the individual corrected degrees of pessimism. The weights are proportional to the corresponding individual degree of absolute risk tolerance. This weighting function is intuitive. Consider a group with two agents having heterogeneous degree of pessimism. Suppose also that the second agent has a lower index of pessimism, but a zero tolerance to risk. Agent 2 will prefer not to gamble in spite of agent 1 offering attractive state prices for high states. Therefore, agent 1 will bear the entire burden of risk. The group's beliefs must therefore rely entirely on agent 1's beliefs. The group's degree of pessimism will be the larger index of pessimism of the first agent. More generally, it is natural that the beliefs of the group to take collective risk decision should be biased in favor of those who bear a larger fraction of this risk, i.e. those who are more risk tolerant. This is the meaning of property (27).

As a direct consequence of the fact that ${ }^{s} \delta^{v}$ is a weighted mean, it is bounded below and above by the smallest and largest individual rate of pessimism:

$$
\min _{\theta \in \Theta}{ }^{s} \delta^{p u}(z, s, \theta) \leq{ }^{s} \delta^{v}(z, s) \leq \max _{\theta \in \Theta}{ }^{s} \delta^{p u}(z, s, \theta),
$$

for all $(z, s)$. It is important to notice that the weight function $T$ is a function of $z$ and $s$. This is made explicit in the notation by indexing the expectation operator $\$$ by $(z, s)$. Thus, even when the individual degrees of pessimism are wealth independent, the societal degree of pessimism needs not be a function of wealth. We examine the relationship between $\delta^{v}$ and $z$ in the next section. 


\section{Wealth effect on the collective degree of pessimism}

In the rest of this paper, we will make the simplifying assumption that individual utility functions are state-independent: $u_{s} \equiv 0$. This has two consequences. First, individual risk tolerances are also independent of the state. Second, the individual degrees of pessimism need not to be corrected by ${ }^{s} \delta^{u}$, and is therefore independent of aggregate wealth. We use this simplifying assumption into the notational shortcut $T^{u}(c, s, \theta)=T^{u}(c, \theta)$ and ${ }^{s} \delta^{p u}(z, s, \theta)={ }^{s} \delta^{p}(s, \theta)$.

Assuming that all members of the group have a multiplicatively separable contribution function $p(s, \theta) u(c, \theta)$ does not imply that the contribution function of the representative agent inherits this property from them. This can be seen from equation (29) where the risk-neutral expectation operator $\mathrm{b}_{z s}$ is wealth-dependent. This implies that the representative agent may have a risk tolerance that depends directly upon the state, or equivalently, that he may have a degree of pessimism that is wealth dependent. These two ways of describing the absence of separability of the contribution function is expressed by the following equality:

$$
-\frac{\partial\left(1 / T^{v}\right)}{\partial s}(z, s)=\frac{\partial^{s} \delta^{v}}{\partial z}(z, s)=\frac{\partial^{2}}{\partial s \partial z} \ln v_{z}(z, s) .
$$

When the contribution is not multiplicatively separable, one can say that the representative agent has a state-dependent utility function, or equivalently, that its subjective probability function is sensitive to change in aggregate wealth.

We start with examining an important special case where such a wealth effect does not exist. It corresponds to situations where the derivative of individual risk tolerances $\partial T^{u} / \partial c$ are all identical and consumption independent. The corresponding set of utility functions is referred to as ISHARA. A utility function has an Harmonic Absolute Risk Aversion (HARA) if its absolute risk tolerance is linear in consumption: $\partial T^{u} / \partial c(c, \theta)=1 / \gamma(\theta)$ for all c. A set of utility functions satisfies the Identically Sloped HARA (ISHARA) property if their absolute risk tolerances are linear in consumption with the same slope: $\gamma(\theta)=\gamma$ for all $\theta$. The set of utility functions that satisfies these 
conditions must be parametrized as follows:

$$
u(c, \theta)=\xi^{\mu} \frac{c-a(\theta)}{\gamma}^{\mathfrak{\emptyset}_{1-\gamma}}
$$

These utility functions are defined over the consumption domain such that $\gamma^{-1}(c-a(\theta))>0$. When $\gamma>0$, parameter $a(\theta)$ is often referred to as the minimum level of subsistence. This preference set includes preferences with heterogeneous exponential utility functions $u(c, t, \theta)=-\exp (-A(\theta) c)$ when $\gamma$ tends to $+\infty$, and $a(\theta) / \gamma$ tends to $-1 / A(\theta)$. Taking $a(\theta)=0$ for all $\theta$, it also includes the set of power (and logarithmic) utility functions with the same relative risk aversion $\gamma$ for all $\theta$.

Proposition 3 Suppose that the individual utility functions are state-independent. The collective degree of pessimism is independent of the wealth per capita in the group $\left(\partial^{s} \delta^{v} / \partial z \equiv 0\right)$ if and only if the members of the group have ISHARA preferences (32).

Proof: Fully differentiating equation (27) with respect to $z$ and using property (23) yields

$$
\left(E T^{u}\right) \frac{\partial^{s} \delta^{v}}{\partial z}(z, s)=\boldsymbol{\Xi}^{s} \delta^{p}(s, \boldsymbol{\vartheta}) \frac{\partial T^{u}}{\partial c}-\left(\boldsymbol{\Xi}^{s} \delta^{u}(s, \boldsymbol{\vartheta})\right)\left(\boldsymbol{\bigotimes} \frac{\partial T^{u}}{\partial c}\right),
$$

where $T^{u}$ and its derivative are evaluated at $(c(z, s, \boldsymbol{\theta}), \boldsymbol{\theta})$, and where $\boldsymbol{\Phi}=\boldsymbol{D}_{z s}$ is the "risk-neutral" expectation operator defined by (30). For ISHARA preferences, $\partial T^{u} / \partial c$ is a constant, which implies that the right-hand side vanishes, and ${ }^{s} \delta^{v}$ is independent of the per capita wealth in the group. To prove necessity, we rewrite condition (33) as

$$
\left(E T^{u}\right) \frac{\partial^{s} \delta^{v}}{\partial z}(z, s)=\emptyset^{s} \delta^{p}(s, \boldsymbol{\theta}) f(z, s, \vartheta)
$$

with

$$
f(z, s, \theta)=\frac{\partial T^{u}}{\partial c}(c(z, s, \theta), \theta)-\mathrm{\phi} \frac{\partial T^{u}}{\partial c}(c(z, s, \boldsymbol{\theta}), \boldsymbol{\theta})^{\lrcorner} .
$$

It implies that the derivative of ${ }^{s} \delta^{v}$ with respect to $z$ is zero for all distributions of $\theta$ only if $f(z, s, \theta)$ is zero uniformly for all $\theta \in \Theta$. From the definition of function $f$, this is the case only if $\partial T^{u} / \partial c$ is independent of $c$ and $\theta$, which means that the group has ISHARA preferences. $¥$ 
The ISHARA condition guarantees that the weights $T^{u} / E T^{u}$ in the expectation operator $\mathbb{D}_{z s}$ remains constant when the wealth level changes in the group. Notice that the ISHARA condition is also necessary for this result. This means that if this condition does not hold, one can find a distribution of individual beliefs in the group such that the collective degree of pessimism depends upon z. In Appendix A, we derive an analytical solution to the aggregation problem when the ISHARA condition is satisfied.

In Proposition 3, we assumed that the derivative of individual absolute risk tolerances and individual degrees of pessimism are independently distributed. In the following Proposition, we alternatively assume that less pessimistic people have a risk tolerance that is more sensitive to changes in consumption. In such a situation, the collective degree of pessimism will be decreasing with the consumption per capita in the economy, in spite of the fact that all consumers have consumption-independent degrees of pessimism.

Proposition 4 Suppose that the individual utility functions are state-independent. The collective degree of pessimism is decreasing with wealth locally in state $(z, s)$ if ${ }^{s} \delta$ and $\partial T^{u} / \partial c$ evaluated respectively at $(s, \boldsymbol{q})$ and $(c(z, s, \boldsymbol{\theta}), \boldsymbol{\theta})$ are anti-comonotone: $\forall\left(\theta, \theta^{\prime}\right) \in \Theta^{2}:\left[{ }^{s} \delta(s, \theta)-{ }^{s} \delta\left(s, \theta^{\prime}\right)\right] \frac{\partial T^{u}}{\partial c}(c(z, s, \theta), \theta)-\frac{\partial T^{u}}{\partial c}\left(c\left(z, s, \theta^{\prime}\right), \theta^{\prime}\right) \leq$ 0 .

Proof: This is a direct consequence of equation (33). $¥$

There is a simple intuition for this result. It comes again from the fact that collective pessimism is a weighted mean of the individual degrees of pessimism. When ${ }^{s} \delta^{p}$ and $\partial T^{u} / \partial c$ are anti-comonotone, an increase in wealth has a larger impact on the weights $T^{u}$ associated to the lower degrees of impatience. An increase in $z$ must then biases ${ }^{s} \delta^{v}$ in favor of less pessimism.

In the special case of utility functions exhibiting constant relative risk aversion (CRRA), viz. $u(c, \theta)=c^{1-\gamma(\theta)} /(1-\gamma(\theta))$, there is a negative relationship between relative risk aversion $\gamma(\theta)$ and $\partial T / \partial c(c, \theta)=1 / \gamma(\theta)$. Thus, the above Proposition applied in the case of CRRA utility functions means that the rate of impatience is a decreasing function of aggregate wealth if less pessimistic agents people are also less risk-averse. The following example illustrates this point. All agents have constant relative risk aversion. The set of state variables is $S=[0,+\infty$ [ with no aggregate risk $(z(s)=z$ for all $s)$. All individual beliefs belong to the negative exponential law with $p(s, \theta)=\theta \exp (-\theta s)$, which implies that ${ }^{s} \delta^{p}(s, \theta)=\theta .{ }^{8}$ There are two types

\footnotetext{
${ }^{8}$ When all beliefs are distributed as $p(s, \theta)=\theta \exp (-\theta s)$, an increase in $\theta$ represents a
} 


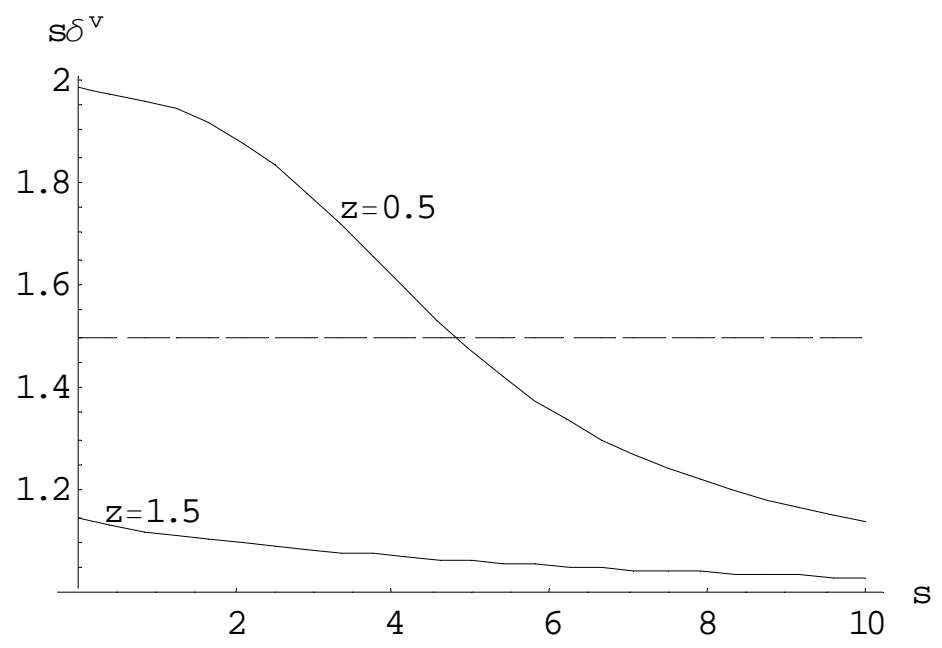

Figure 1: The collective degree of pessimism for two different wealth level when individual degrees of pessimism and relative risk aversion are both constant and are negatively correlated. The values of the parameters are $\theta_{p}=2, \theta_{o}=1, \gamma_{p}=1$ and $\gamma_{o}=10$.

of agents. The pessimistic type $p$ has a degree of pessimism $\theta_{p}$ and a constant risk aversion $\gamma_{p}$, whereas the optimistic type $o$ has a lower degree of pessimism $\theta_{o}<\theta_{p}$ and a higher degree of risk aversion $\gamma_{o}>\gamma_{p}$. In Figure 1, we have drawn the collective degree of pessimism for the efficient allocation associated to Pareto-weights with $\lambda\left(\theta_{p}\right) / \lambda\left(\theta_{o}\right)=\theta_{0} / \theta_{p}$, respectively when the group's wealth per capita equals $z=0.5$ and $z=1.5$. We see that the poorer group is more pessimistic at the collective level.

\section{The effect of increasing disagreement on the collective degree of pessimism}

We establish here the link between the collective degree of pessimism characterized by ${ }^{s} \delta^{v}$ and the heterogeneity of individual indexes of pessimism. In order to focus on the heterogeneity of beliefs, we assume in this section that risk preferences are homogenous: $\partial T^{u} / \partial \theta \equiv 0$. In a given group, we compare

uniform increase in pessimism in the sense of Abel (2002). 
states in the neighborhood of $s \in S$, with the same wealth per capita $z$, but with a different distribution of individual subjective state probabilities. In this section, we first define the notion of increasing disagreement, which basically means that the distribution of the state probability across agents becomes more dispersed when comparing increasing $s \in S$. We then show how the increase in disagreement affect the collective degree of pessimism.

\subsection{Increasing disagreement}

We consider the distribution of $q(s, \boldsymbol{\theta})=\lambda(\boldsymbol{\theta}) p(s, \boldsymbol{\theta})$ where $q(s, \theta)$ denotes the Pareto-weighted subjective probability of state $s$ by agent $\theta$. The cakesharing problem (19) can then be rewritten as

$$
v(z, s)=\max _{c^{0}(z,, \theta)} E^{\mathrm{h}} q(s, \boldsymbol{\theta}) u(c(z, s, \boldsymbol{\theta}))^{\mathrm{i}} \quad \text { s.t. } E c(z, s, \boldsymbol{\theta})=z .
$$

Suppose without loss of generality that $q(s, \theta)$ is nonincreasing in $\theta$ for the state $s$ under consideration. We define the measure of disagreement as

$$
{ }^{\theta} \delta^{q}(s, \theta)=-\partial \ln q(s, \theta) / \partial \theta \geq 0 .
$$

We assume that, in the neighborhood of $s$, larger states correspond to more disagreement. In other words, we assume that $q(s, \boldsymbol{\theta})$ has a more dispersed distribution when $s$ increases. Using $\partial^{\theta} \delta^{q} / \partial s=\partial^{s} \delta^{q} / \partial \theta$, we have the following definition.

Definition 1 Consider a specific state of nature s, and define types in such a way that $q_{\theta}(s, \theta) \leq 0$ for all $\theta \in \Theta$. We say that disagreement is locally increasing in $s$ if ${ }^{\theta} \delta^{q}(s, \theta)$ is increasing in $s$. This is equivalent to require that $q$ satisfies the Monotone Likelihood Ratio (MLR) property that ${ }^{s} \delta^{q}(s, \theta)$ is increasing in $\theta$, or that $q(s, \theta) / q\left(s, \theta^{\prime}\right)$ is increasing in $s$ whenever $\theta<\theta^{\prime}$.

In other words, $q(s, \theta)$ and ${ }^{s} \delta^{q}(s, \theta)$ must be anti-comonotone in $\theta$ :

$$
\frac{\partial^{s} \delta^{p}(s, \theta)}{\partial \theta} \frac{\partial}{\partial \theta} \ln q(s, \theta)=-\frac{\partial^{s} \delta^{p}(s, \theta)}{\partial \theta}{ }^{\theta} \delta^{q}(s, \theta) \leq 0 .
$$

Disagreement is increasing in $s$ because high types $\theta$ have a low $q$ which is decreasing relatively more quickly with $s$. Thereby, it amplifies the dispersion of $q(s, \boldsymbol{\theta})$. Figure ?? describes such a situation. Notice that our definition of 


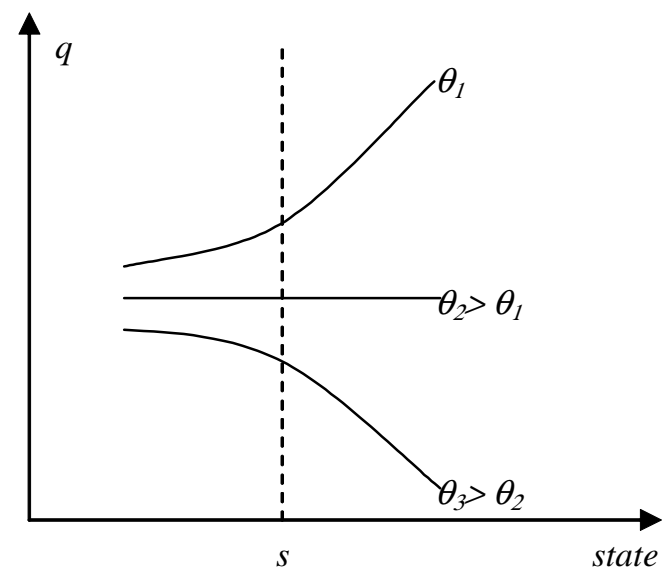

Figure 2: In the neighborhood of $s$, disagreement on the Pareto-weighted state probability is increasing with the state.

increasing disagreement does not constrain in any way how the mean state probability fluctuates across states.

The dispersion of $q(s, \boldsymbol{\theta})$ is related to consumption inequalities in state $s$. Using the equivalence with the individual portfolio problem (1) and more particularly equation $(7)$, we can verify that

$$
c_{\theta}(z, s, \theta)=-{ }^{\theta} \delta^{q}(s, \theta) T^{u}(c(z, s, \theta))
$$

The absolute value of $c_{\theta}(z, s, \theta)$ is a local measure of the degree of consumption inequality in state $(z, s)$. This equation explains why we use ${ }^{\theta} \delta^{q}$ rather than ${ }^{\theta} \delta^{p}$ to measure disagreement. Indeed, it is differences in $q$ rather than differences in $p$ which explains the inequality in the distribution of consumption. This distribution of individual consumption levels and its subsequent effect on risk tolerances are the driving forces behind the determination of the collective degree of pessimism. 


\subsection{Comparison of collective pessimism with the mean individual degree of pessimism}

In an homogenous economy, all agents have the same degree of pessimism ${ }^{s} \delta^{p}$ which are perfectly in line with the collective degree of pessimism ${ }^{s} \delta^{v}$. In this subsection, we examine the effect of disagreement on collective pessimism. More specifically, we characterize the conditions under which ${ }^{s} \delta^{v}(z, s)$ is smaller than $E^{s} \delta^{p}(s, \vartheta)$. As explained earlier, this is what matters to relate individual rules to collective rules for portfolio choices and asset pricing. We will show that the relationship between ${ }^{s} \delta^{v}$ and $E^{s} \delta^{p}$ depends upon whether we consider a region of states with increasing or decreasing disagreement.

Let ${ }^{s} \bar{\delta}^{p}(z, s)=E^{s} \delta^{p}(s, \vartheta)$ denote the mean individual degree of pessimism. Using property (27), we want to determine the condition under which

$$
s \delta^{v}(z, s)=\frac{E^{s} \delta^{p}(s, \boldsymbol{\theta}) T^{u}(c(z, s, \boldsymbol{\vartheta}))^{\mathbf{i}}}{E T^{u}(c(z, s, \boldsymbol{\theta}))} \leq E^{s} \delta^{p}(s, \boldsymbol{\theta})={ }^{s} \bar{\delta}^{p}(s) \text {. }
$$

The simplest case arises when all agents in the heterogeneous group have the same utility function with constant absolute risk aversion (CARA). This is the only case guaranteeing that weights $T^{u} / E T^{u}$ in equation (37) are uniformly equal to unity, which implies that ${ }^{s} \delta^{v}(z, s)={ }^{2} \bar{\delta}^{p}(s)$ for all $(z, s)$. The heterogeneity of beliefs has no effect on the collective degree of pessimism in that case.

Condition (37) can be rewritten as

$$
E^{s} \delta^{p}(s, \vartheta)=\bar{\delta} \Longrightarrow E^{\mathrm{h}} \delta^{p}(s, \boldsymbol{\vartheta}) T^{u}(c(z, s, \boldsymbol{\vartheta}))^{\mathrm{i}} \leq \bar{\delta} E^{\mathrm{h}} T^{u}(c(z, s, \boldsymbol{\vartheta}))^{\mathrm{i}} \text {. }
$$

The following Lemma is useful to solve this kind of problem.

Lemma 1 Consider two functions $f_{1}$ and $f_{2}$ from $R$ to $R^{++}$, and $g: R \rightarrow R$. Then, the following two conditions are equivalent:

1. For any random variable $\theta$ and for any constant $\bar{g}, E f_{1}(\theta) g(\boldsymbol{\theta})=$ $\bar{g} E f_{1}(\boldsymbol{\theta})$ implies that $E f_{2}(\boldsymbol{\theta}) g(\boldsymbol{\theta}) \leq \bar{g} E f_{2}(\boldsymbol{\theta})$.

2. $f_{1}(\theta) / f_{2}(\theta)$ and $g(\theta)$ are comonotone. 
Proof: It is a direct consequence of the "diffidence theorem" in Gollier and Kimball (1996) (Proposition 11 in Gollier (2001)). $¥$

Let us define types in such a way that $q_{\theta}(s, \theta) \leq 0$ for all $\theta \in \Theta$. We can apply this Lemma where its statement 1 corresponds to condition (38) with $f_{1}(\theta)=1, f_{2}(\theta)=T^{u}(c(z, s, \theta))$ and $g(\theta)={ }^{s} \delta^{p}(s, \theta)$ for all $\theta$. Because we assume that $s$ is in the region of increasing disagreement, function $g$ is increasing in $\theta$. Thus, statement 2 in the Lemma requires that $A^{u}(c(z, s, \theta))=\left(T^{u}(c(z, s, \theta))\right)^{-1}$ be increasing in $\theta$. Because $q_{\theta} \leq 0$, equation (36) tells us that $c$ is decreasing in $\theta$. We conclude that condition (38) is satisfied if and only if $A^{u}$ is decreasing in $c$. This result is summarized in the following Proposition.

Proposition 5 Suppose that the individual utility functions are identical and state-independent. Consider a state $s \in S$ around which there is increasing disagreement. The following two conditions are equivalent:

1. The collective degree of pessimism ${ }^{s} \delta^{v}(z, s)$ is smaller than the mean individual degree of pessimism ${ }^{s} \bar{\delta}^{p}(s)=E^{s} \delta^{p}(s, \vartheta)$ for all distributions of $\theta$;

2. The absolute risk tolerance $T^{u}(c)$ is increasing in $c(D A R A)$.

An increase in disagreement that maintains the mean degree of pessimism reduces collective pessimism if agents have decreasing absolute risk aversion. This property is reversed under increasing absolute risk aversion. Observe that these results describe properties that are purely local in a neighborhood of $s$ with increasing or decreasing disagreement. In the next Proposition, we take a more global approach by assuming that the MLR condition holds for all $s \in S$.

Proposition 6 Suppose that all agents have the same state-independent utility function that exhibits decreasing absolute risk aversion. Suppose also that the $M L R$ condition $\partial^{s} \delta^{q} / \partial \theta \geq 0$ holds for all $s \in S$, and that there exists a state $t \in S$ such that $q(t, \theta)=\bar{q}$ for all $\theta \in \Theta$. It implies that, for all $z$, the collective degree of pessimism ${ }^{s} \delta^{v}(z, s)$ crosses the mean degree of pessimism $s \bar{\delta}^{p}(s)$ only at $t$, from above.

Proof: By assumption, $q_{\theta}(s, \theta)=0$ for all $\theta$ at $s=t$. It implies by (36) that $c_{\theta}(z, t, \theta)=0$ for all $\theta$. This implies in turn from $(27)$ that ${ }^{s} \delta^{v}(z, t)={ }^{s}$ 
$\bar{\delta}^{p}(t)$. Using Proposition 5, we just need to prove that $q_{\theta}$ is negative for all $s$ larger than $t$, and that it is positive for all $s$ smaller than $t$. Observe that the MLR condition $\partial^{s} \delta^{q} / \partial \theta \geq 0$ implies that $q_{\theta s} \leq 0$ at any $s$ were $q_{\theta}=0$. It implies that the $q_{\theta}$ curve can cross the horizontal $s$-axis only once at $t$ for all $\theta$, from above. $¥$

To illustrate, let us consider a group with DARA in which all agents' expectations are representable by negative exponential laws: $p(s, \theta)=\theta \exp (-\theta s)$. This implies that ${ }^{s} \delta^{q}(s, \theta)=\theta$. Let us consider the Pareto-efficient risk sharing which corresponds to $\lambda\left(\theta_{p}\right)=\lambda\left(\theta_{o}\right)$. There are two types of agents respectively with degree of pessimism $\theta_{p}$ and $\theta_{o} \leq \theta_{p}$. It yields a crossing state $t=\left(\log \theta_{p}-\log \theta_{o}\right) /\left(\theta_{p}-\theta_{o}\right)$. From any state $s$, selecting another state in the direction opposite to $t$ yields more disagreement. The above Proposition states that the heterogeneity of beliefs yields relatively more pessimistic in low states, and relatively less pessimistic in high states. Figure ?? illustrates this case with $u(c)=c^{0.9}, \theta_{o}=1$ and $\theta_{p}=2$.

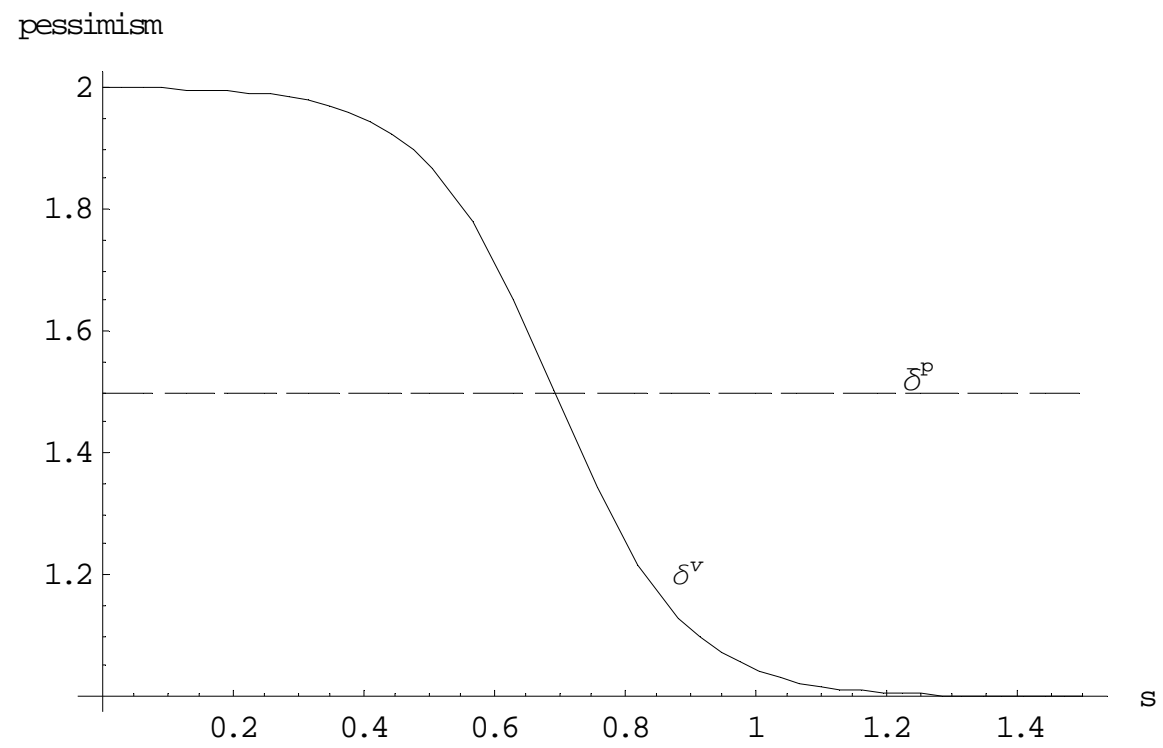

Effect of disagreement on the collective degree of pessimism.

What insights does it bring for the collective risk management? Suppose that the reference rule for the collective decision maker is to estimate the individual ${ }^{s} \delta^{q}$. From the distribution of the individual degrees of pessimism, the decision-maker extracts the mean. This mean ${ }^{s} \bar{\delta}^{p}$ is then used to select the optimal collective risk exposure by using the rules presented in section 2. Suppose for example that the group faces state-prices $\pi($.$) such that$ 
${ }^{s} \delta^{\pi}(s)={ }^{s} \bar{\delta}^{p}(s)$. From his computation of ${ }^{s} \bar{\delta}^{p}$, market prices are actuarially fair. Following portfolio rule (7), it implies that the planner recommends the group to fully insure their collective risk $\left(c_{s}=0\right)$. By doing so, the decision-maker overlooks the fact that the heterogeneity in the distribution of pessimism in the population induces people to speculate on the states that they believe are relatively more likely to occur. Because those bearing risk should receive a larger weight in the mean, this reference rule is not efficient. When the MLR condition is satisfied, Proposition 6 states that the decisionmaker overestimates the collective degree of pessimism in the region of $S$ with increasing disagreement. By equation (7), his efficient net demand for ArrowDebreu securities should be decreasing in $s$ in this subset of $S$. Reciprocally, the efficient state consumption per capita should be decreasing in $s$ in the region of $S$ with decreasing disagreement. In the example presented above, it means that the socially efficient consumption per capita is U-shaped with respect to $s$.

One can translate this results on collective pessimism into their equivalent effect on collective subjective probabilities. Let $\emptyset$ denote the probability distribution that yields a degree of pessimism ${ }^{s} \bar{\delta}^{p}$, i.e., $-\boldsymbol{D}_{s}(s) / \mathbb{D}(s)={ }^{s} \bar{\delta}^{p}(s)$ for all $s$. This would the implicit density function that the planner would take under the reference decision rule. We compare it to the efficient density function $p^{v}(z, s)=v_{z}(z, s) /{ }_{S} v_{z}(z(t), t) d t$. Compared to $\boldsymbol{D}$, the heterogeneous group uses collective subjective density $p^{v}$ in which some probability mass is taken away from an interval in the center of the support of states to be transferred to the extreme states, as seen in Figure 3 for the example presented above. Indeed, the single-crossing property exhibited by $\left({ }^{s} \bar{\delta}^{p},{ }^{s} \delta^{v}\right)$ generates a double-crossing property in the density functions.

Corollary 1 Suppose that all agents have the same state-independent utility function that exhibits decreasing absolute risk aversion. Suppose also that the $M L R$ condition $\partial^{s} \delta^{q} / \partial \theta \geq 0$ holds for all $s \in S$, and that there exists a state $t \in S$ such that $q(t, \theta)=\bar{q}$ for all $\theta \in \Theta$. It implies that, for all $z$, there exists an interval $I_{z} \subset S$ containing $t$ such that

$$
p^{v}(z, s) \quad \begin{aligned}
& 1 / 2 \\
& \geq \mathfrak{D}(s) \quad \text { if } \quad s \in I_{z} \\
& \geq(s) \quad \text { if } \quad s \notin I_{z},
\end{aligned}
$$

where the probability densities $p^{v}$ and $₫$ are characterized respectively by $-p_{s}^{v}(z, s) / p^{v}(z, s)={ }^{s} \delta^{v}(z, s)$ and $-\mathfrak{b}(s) / \mathfrak{b}(s)={ }^{s} \bar{\delta}^{p}(s)$. These inequalities are reversed under increasing absolute risk aversion. 


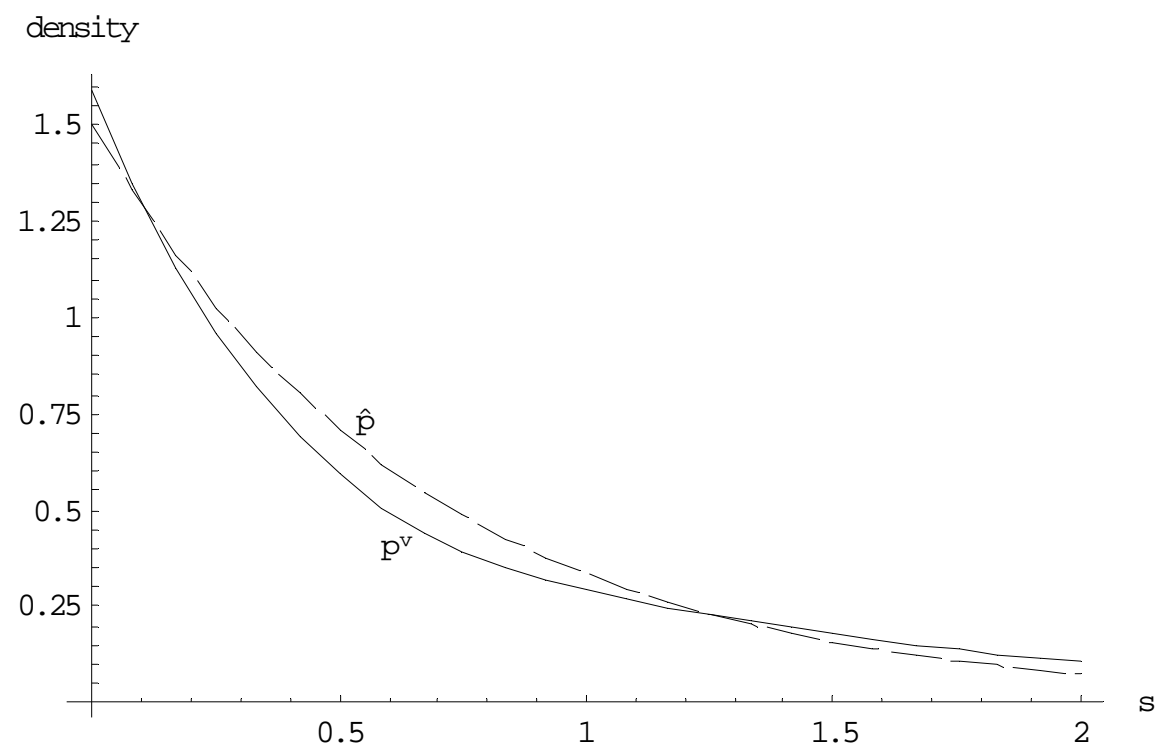

Figure 3: Effect of disagreement on the collective probability density.

Proof: Consider a specific $z$. Suppose that there exists a $t=t(z)$ such that ${ }^{s} \delta^{v}(z,$.$) and { }^{s} \bar{\delta}^{p}($.$) cross at t$. It implies from proposition 6 that

$$
-\frac{p_{s}^{v}(z, s)}{p^{v}(z, s)} \geq-\frac{\boldsymbol{p}_{s}(s)}{\mathfrak{\emptyset}(s)}
$$

for all $s \leq t$. It implies in turn that there can exist at most one $t_{1}=t_{1}(z) \leq t$ such that $p^{v}\left(z, t_{1}\right)=\mathbf{p}\left(t_{1}\right)$. Moreover, at $s=t_{1}$, the above inequality implies that $p_{s}^{v}\left(z, t_{1}\right) \leq \boldsymbol{p}_{s}\left(t_{1}\right)$. Similarly,Proposition 6 implies that

$$
-\frac{p_{s}^{v}(z, s)}{p^{v}(z, s)} \leq-\frac{\boldsymbol{p}_{s}(s)}{\mathfrak{p}(s)}
$$

for all $s \geq t$. It implies that there can exist at most one $t_{2}=t_{2}(z) \geq t$ such that $p^{v}\left(z, t_{2}\right)=\mathfrak{\emptyset}\left(t_{2}\right)$, with $p_{s}^{v}\left(z, t_{2}\right) \geq \emptyset\left(t_{2}\right)$. This concludes the proof, with $I_{z}=\left[t_{1}, t_{2}\right]$. $¥$ 


\subsection{Comparison of collective pessimism with the probability- weighted mean of individual degrees of pessimism}

In the reference aggregation rule that we considered above, we assumed that the planner first estimates individual degrees of pessimism ${ }^{s} \delta^{p}(., \vartheta)$ from which he computes the mean ${ }^{s} \bar{\delta}^{p}($.$) . We showed how this miscalibration af-$ fects the socially efficient collective risk exposure. In this section, we consider an alternative aggregation procedure for the planner. Namely, we assume that the planner first estimates the individual subjective probabilities $p(., \boldsymbol{\theta})$ from which he computes the Pareto-weighted mean $\vec{p}()=.E \lambda(\boldsymbol{\theta}) p(., \boldsymbol{\vartheta})$. He then estimates an aggregate degree of pessimism by computing $s \vec{\delta}^{p}()=$. $\vec{p}_{s}(.) / \vec{p}($.$) in order to apply the portfolio rules described in section 2$ at the collective level. This aggregation rule yields policy recommendations that differ from those obtained with the reference aggregation rule. We examine in this subsection the miscalibration of such alternative aggregation procedure.

We want to determine the conditions under which ${ }^{s} \delta^{v}(z, s)$ is smaller than

$$
s \vec{\delta}^{p}(s)=-\frac{E \lambda(\boldsymbol{\vartheta}) p_{s}(s, \boldsymbol{\vartheta})}{E \lambda(\boldsymbol{\vartheta}) p(s, \boldsymbol{\vartheta})}=\frac{E q(s, \boldsymbol{\vartheta})^{s} \delta^{p}(s, \boldsymbol{\vartheta})}{E q(s, \boldsymbol{\vartheta})} .
$$

Observe that ${ }^{s} \vec{\delta}^{p}$ is a probability-weighted mean of individual degrees of pessimism. We can use Lemma 1 by observing that ${ }^{s} \delta^{v}(z, s)$ is smaller than $s \vec{\delta}^{p}(s)$ if

$E q(s, \vartheta)^{s} \delta^{p}(s, \vartheta)=\vec{\delta} E q(s, \vartheta) \Longrightarrow E^{s} \delta^{p}(s, \vartheta) T^{u}(c(z, s, \vartheta))^{\mathbf{i}} \leq \vec{\delta} E^{\mathrm{h}} T^{u}(c(z, s, \vartheta))^{\mathbf{i}}$.

Before presenting this result, it is noteworthy that condition ${ }^{s} \delta^{v}(z, s) \leq$ $s \vec{\delta}^{p}(s)$ is more demanding than ${ }^{s} \delta^{v}(z, s) \leq{ }^{s} \bar{\delta}^{p}(s)$ in the region of increasing disagreement. This is because ${ }^{s} \vec{\delta}^{p}(s)$ is smaller than ${ }^{s} \bar{\delta}^{p}(s)$ in this region. This is due to the fact that increasing disagreement is equivalent to the assumption that $q(s, \theta)$ and ${ }^{s} \delta^{p}(s, \theta)$ are anti-comonotone in $\theta$, which implies that

$$
E q(s, \vartheta)^{s} \delta^{p}(s, \vartheta) \leq E q(s, \vartheta) E^{s} \delta^{p}(s, \vartheta) .
$$

This observation induces that the restriction on preferences that guarantees the comparative statics property will be stronger than decreasing absolute risk aversion $\left(T_{c}^{u}(c) \geq 0\right.$ for all $\left.c\right)$. 
Proposition 7 Suppose that the individual utility functions are identical and state-independent. Consider a state $s \in S$ around which there is increasing disagreement. The following two conditions are equivalent:

1. The collective degree of pessimism ${ }^{s} \delta^{v}(z, s)$ is smaller than the probabilityweighted mean of individual degrees of pessimism ${ }^{s} \vec{\delta}^{p}(s)=E q(s, \vartheta)^{s} \delta^{p}(s, \vartheta) / E q(s, \vartheta)$ for all distributions of $\theta$;

2. The derivative of absolute risk tolerance with respect to consumption is larger than unity: $T_{c}^{u}(c) \geq 1$ for all $c$.

Proof: We use Lemma 1 with $f_{1}(\theta)=q(s, \theta), f_{2}(\theta)=T^{u}(c(z, s, \theta))$ and $g(\theta)={ }^{s} \delta^{p}(s, \theta)$. Using the first-order condition associated to program (19), we obtain that $f_{1}(\theta)=\psi(z, s) / u^{\prime}(c(z, s, \theta))$, where $\psi(z, s)$ is the Lagrange multiplier associated to the feasibility constraint. Lemma 1 requires that $f_{1}(\theta) / f_{2}(\theta)=\psi(z, s) /\left[u^{\prime}(c(z, s, \theta)) T^{u}(c(z, s, \theta))\right]$ and ${ }^{s} \delta^{p}(s, \theta)$ be comonotone in $\theta$. Because we consider the case of increasing disagreement, we can assume without loss of generality that $q$ is decreasing in $\theta$ and that ${ }^{s} \delta^{p}(s, \theta)$ is increasing in $\theta$. By property (36), the first assumption implies that $c(z, s, \theta)$ is decreasing in $\theta$. It implies that $f_{1} / f_{2}$ and $g$ are comonotone if and only if $u^{\prime}(c) T^{u}(c)$ is increasing in $c$. This is the case if

$$
u^{\prime \prime}(c) T^{u}(c)+u^{\prime}(c) T_{c}^{u}(c)=u^{\prime \prime}(c) T^{u}(c)\left[1-T_{c}^{u}(c)\right] \geq 0
$$

for all $c$. This is equivalent to require that $T_{c}^{u}(c) \geq 1$. $¥$

We can estimate the deviation of ${ }^{s} \delta^{v}(z, s)$ with respect to ${ }^{s} \vec{\delta}^{p}(s)$ if we assume that the divergence of opinions is small. Observe that the difference between the two aggregate measures of pessimism can be measured by a difference in covariance:

$s \vec{\delta}^{p}(s)-{ }^{s} \delta^{v}(z, s)=\frac{\operatorname{cov}\left({ }^{s} \delta^{p}(s, \vartheta), 1 / u^{\prime}(c(z, s, \vartheta))\right)}{E 1 / u^{\prime}(c(z, s, \vartheta))}-\frac{\operatorname{cov}\left({ }^{s} \delta^{p}(s, \vartheta), T^{u}(c(z, s, \vartheta))\right)}{E T^{u}(c(z, s, \boldsymbol{\vartheta}))}$.

If $\theta$ has a support in a small neighborhood of $\theta_{0}$, using Taylor expansions yields the following approximation:

$$
\begin{aligned}
s \vec{\delta}^{p}(s)-{ }^{s} \delta^{v}(z, s) & \simeq\left(T_{c}^{u}(c)-1\right)^{\theta} \delta^{q}\left(s, \theta_{0}\right) \frac{\partial^{s} \delta^{p}\left(s, \theta_{0}\right)}{\partial \theta} \sigma_{\theta}^{2} \\
& =\left(1-T_{c}^{u}(c)\right) \operatorname{cov}\left(\ln q(s, \boldsymbol{\theta}),{ }^{s} \delta^{p}(s, \boldsymbol{\theta})\right)
\end{aligned}
$$


with $c=c\left(z, s, \theta_{0}\right)$. In the region of increasing disagreement, the sign of the right-hand side of this approximation is positive if and only if $T_{c}^{u}(c)$ is larger than unity, as stated by Proposition 7. Observe that the deviation of ${ }^{s} \delta^{v}(z, s)$ with respect to ${ }^{s} \vec{\delta}^{p}(s)$ does not depend upon the degree of disagreement. It is approximately proportional to the rate of increase of disagreement which is measured by $-\operatorname{cov}\left(\ln q(s, \vartheta),{ }^{s} \delta^{p}(s, \vartheta)\right)$.

Considering the particular case in which $E \lambda(\boldsymbol{\theta}) p_{s}(s, \boldsymbol{\theta})=0$, or equivalently ${ }^{s} \vec{\delta}^{p}(s)=0$, we obtain the following Corollary which is due to Varian (1985) and Ingersoll (1987).

Corollary 2 Suppose that the individual utility functions are identical and state-independent. Consider an interval in $S$ in which increasing s increases disagreement but preserves the Pareto-weighted mean subjective probability $E \lambda(\boldsymbol{\theta}) p(s, \boldsymbol{\theta})$. If $T_{c}^{u}(c) \geq 1$ for all $c$, then the collective subjective probability $p^{v}(z, s)$ is increasing in $s$ in this interval.

Notice that

$$
T_{c}^{u}(c)=\frac{P^{u}(c)}{A^{u}(c)}-1 \text { with } A^{u}(c)=-\frac{u^{\prime \prime}(c)}{u^{\prime}(c)} \text { and } P^{u}(c)=-\frac{u^{\prime \prime \prime}(c)}{u^{\prime \prime}(c)} .
$$

$A^{u}$ and $P^{u}$ are respectively the degree of absolute risk aversion and absolute prudence. Kimball (1990) shows that absolute prudence is useful to measure the impact of risk on the marginal value of wealth. Namely, he shows that the effect of risk on the marginal value of wealth is equivalent to a sure reduction of wealth that is approximately proportional to the product of the variance of the risk by $P^{u}$. Using equation (44), the derivative of absolute risk tolerance is larger than unity if and only if absolute prudence is larger than twice the absolute risk aversion:

$$
T_{c}^{u}(c) \geq 1 \Leftrightarrow P^{u}(c) \geq 2 A^{u}(c) .
$$

There is a simple intuition to Corollary 2. It states that, everything else unchanged, the group should devote more effort to finance aggregate consumption in states with more disagreement if $P^{u}$ is larger than $2 A^{u}$. The paradigm of the veil of ignorance is useful for this intuition. Under the veil of ignorance, more disagreement can be reinterpreted as more risk, which has two conflicting effects on the marginal value of aggregate wealth $v_{z}$. The first effect is a precautionary effect. The increase in risk has an 
effect on $v_{z}$ that is equivalent to a sure reduction of aggregate wealth that is approximately proportional to absolute prudence. But this does not take into account of the fact that the group does rebalance consumption towards the more optimistic agents. This endogenous negative correlation between the weighted probability $\lambda(\theta) p(s, \theta)$ and individual consumption is favorable to the expected consumption $E \lambda(\boldsymbol{\theta}) p(s, \boldsymbol{\theta}) c(z, s, \boldsymbol{\vartheta})$. Under the veil of ignorance, this makes the representative agent implicitly wealthier, thereby reducing the marginal value of wealth. This wealth effect is proportional to the rate at which marginal utility decreases with consumption. It is thus proportional to $A^{u}$. Globally, more disagreement raises the marginal value of wealth if the precautionary effect dominates the wealth effect, or if absolute prudence is sufficiently larger than absolute risk aversion. This provides an intuition to condition $P^{u} \geq 2 A^{u}$, or $T_{c}^{u} \geq 1 .^{9}$

The reader can easily obtain the following results by symmetry with what we obtained in the previous subsection.

Proposition 8 Suppose that all agents have the same state-independent utility function that exhibits the property that $T_{c}^{u} \geq 1$. Suppose also that the $M L R$ condition $\partial^{s} \delta^{q} / \partial \theta \geq 0$ holds for all $s \in S$, and that there exists a state $t \in S$ such that $q(t, \theta)=\bar{q}$ for all $\theta \in \Theta$. It implies that, for all $z$, the collective degree of pessimism ${ }^{s} \delta^{v}(z, s)$ crosses the mean degree of pessimism ${ }^{s} \vec{\delta}^{p}(s)$ only at $t$, from above.

Corollary 3 Suppose that all agents have the same state-independent utility function that exhibits the property that $T_{c}^{u} \geq 1$. Suppose also that the $M L R$ condition $\partial^{s} \delta^{q} / \partial \theta \geq 0$ holds for all $s \in S$, and that there exists a state $t \in S$ such that $q(t, \theta)=\bar{q}$ for all $\theta \in \Theta$. It implies that, for all $z$, there exists an interval $I_{z} \subset S$ containing $t$ such that

$$
\begin{aligned}
p^{v}(z, s)^{1 / 2} & \leq \vec{p}(s) \quad \text { if } \quad s \in I_{z} \\
& \geq \vec{p}(s) \quad \text { if } \quad s \notin I_{z},
\end{aligned}
$$

where the probability densities $p^{v}$ and $\vec{p}$ are characterized respectively by $-p_{s}^{v}(z, s) / p^{v}(z, s)={ }^{s} \delta^{v}(z, s)$ and $\vec{p}(s)=E \lambda(\boldsymbol{\vartheta}) p(s, \boldsymbol{\vartheta})$. These inequalities are reversed under increasing absolute risk aversion.

\footnotetext{
${ }^{9}$ In the Arrow-Debreu portfolio context, Gollier (2002) shows that condition $P^{\mathrm{u}} \geq 2 A^{\mathrm{u}}$ is necessary and sufficient for a mean-preseving spread in the distribution of state price per unit of probability to raise the marginal value of wealth.
} 
These results are reversed if $T_{c}^{u}$ is less than unity.

The assumption that agents have decreasing absolute risk aversion is a widely accepted hypothesis in our profession. The plausibility of condition $T_{c}^{u} \geq 1$ is much more questionable. In fact, most specialists in the field believe that $T_{c}^{u}$ is smaller than unity. The argument goes as follows. In the absence of any direct estimate of the sensitivity of absolute risk tolerance to changes in wealth, we consider the CRRA specification $u(c)=c^{1-\gamma} /(1-\gamma)$ for which $T^{u}(c)=c / \gamma$. It implies that $T_{c}^{u} \geq 1$ if and only if $\gamma$ is smaller than unity. Relying on asset pricing data and the equity premium puzzle, one must conclude that relative risk aversion must be much larger than unity. Therefore, all results in this subsection should be interpreted by considering their contraposition with $T_{c}^{u}(c) \leq 1$ ! Notice that the limit case is the logarithmic utility function for which $T_{c}^{u}(c) \equiv 1$. This is the only case in which using the alternative aggregation rule based on $\vec{p}(s)=E \lambda(\vartheta) p(s, \vartheta)$ is socially efficient. When $T_{c}^{u}(c) \leq 1$, using this rule will induce the planner to demand too many Arrow-Debreu securities in states with a low level of disagreement, and not enough in states with a high level of disagreement. This conclusion is exactly opposite to the one presented in the previous subsection when the planner use the aggregation rule based on ${ }^{s} \bar{\delta}^{p}$.

One of the consequences of this result is that $p^{v}(z, s)$ needs not be in the interval bounded by $\min _{\theta \in \Theta} p(s, \theta)$ and $\max _{\theta \in \Theta} p(s, \theta)$. Consider the special case of equal Pareto weights $\lambda(\theta)=1$ for all $\theta$. Suppose that there is a state $t$ for which there is no divergence of opinion about the probability $p(t, \theta)=\vec{p}$ of occurrence. Using Corollary 3 with the plausible $T_{c}^{u} \leq 1$, it implies that the collective probability $p^{v}(\mathbf{e}, t)$ of state $t$ is larger than the unanimously agreed individual probability of that state! More generally, the state probability of the representative agent may not be within the range of the probabilities associated to that state by the different agents of the group. This is in sharp contrast with the collective degree of pessimism, which we know is a weighted average of individual degrees of pessimism.

We illustrate these results by the following example. There is a continuum of states represented by $S=[0,1]$. There are two agents in the group. The beliefs of agent $\theta_{p}$ are represented by the density function $p\left(s, \theta_{p}\right)=2-2 s$, which implies that ${ }^{s} \delta^{p}\left(s, \theta_{p}\right)=(1-s)^{-1}$. Agent $\theta_{o}$ has a density function $p\left(s, \theta_{o}\right)=2 s$, which implies that ${ }^{s} \delta^{p}\left(s, \theta_{o}\right)=-s^{-1}$. Observe that the MLR condition is satisfied for this pair of beliefs. They are represented by the dashed lines in Figure 5. We assume that agents have the same state- 


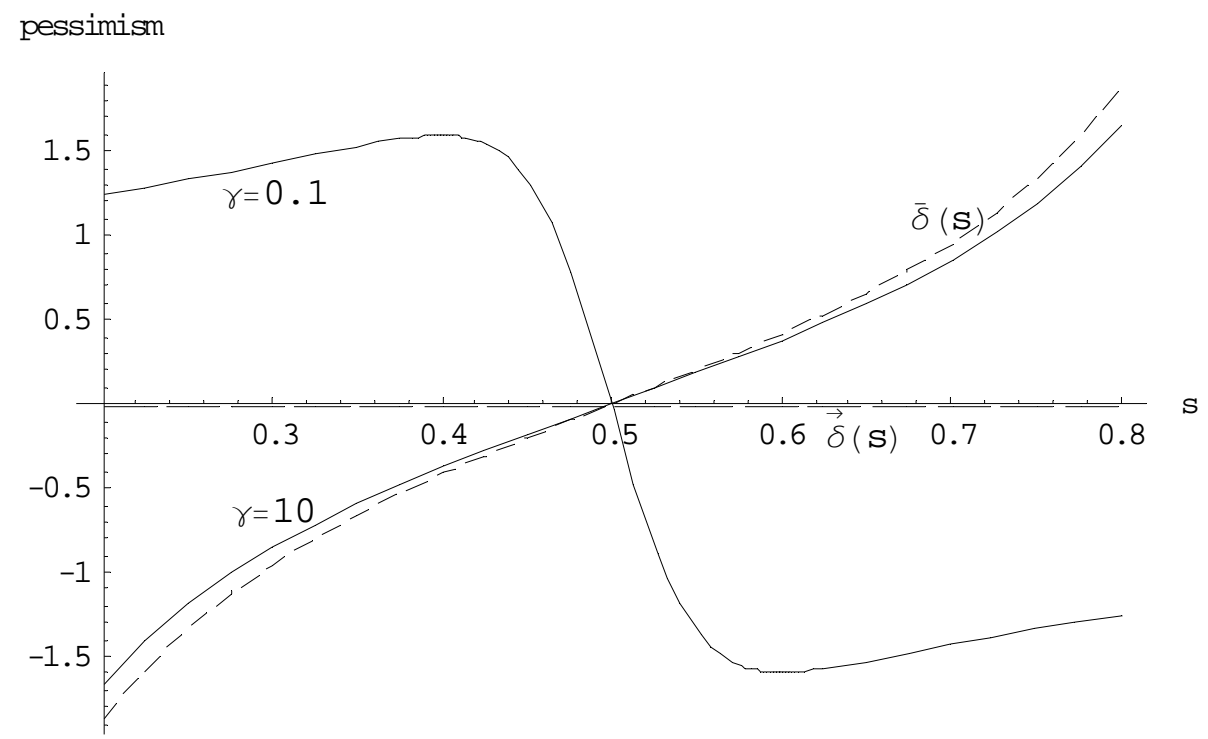

Figure 4: The collective degree of pessimism for $u(c, \theta)=c^{1-\gamma} /(1-\gamma)$, $\lambda\left(\theta_{p}\right)=\lambda\left(\theta_{o}\right), p\left(s, \theta_{p}\right)=2-2 s$, and $p\left(s, \theta_{o}\right)=2 s$.

independent utility function $u(c)=c^{1-\gamma} /(1-\gamma)$, where $\gamma$ is constant relative risk aversion. We consider the Pareto efficient allocation associated to $\lambda\left(\theta_{p}\right)=\lambda\left(\theta_{o}\right)$. Increasing $s$ from 0 first tends to reduce disagreement. The index of disagreement goes to zero for state $t=0.5$. Above $t$, increasing $s$ increases disagreement. Because $E \lambda(\boldsymbol{\theta}) p(s, \boldsymbol{\vartheta})=1$ for all $s \in S$, we immediately obtain that ${ }^{s} \vec{\delta}^{p}(s) \equiv 0$. We draw Figure 4 where the two plain curves are for the efficient collective degrees of pessimism ${ }^{s} \delta^{v}$, respectively for $\gamma=10$ and $\gamma=0.1$. We see that ${ }^{s} \delta^{v}$ and ${ }^{s} \vec{\delta}^{p} \equiv 0$ cross only once respectively from below and from above, as stated in Proposition 8. We also observe that the curve representing the efficient collective degree of pessimism always cross $s \bar{\delta}^{p}$ (dashed curve) from above, because $u$ exhibits DARA. This is another illustration of Proposition 6.

Observe that because the mean state density is uniformly equal to unity, we can apply Corollary 2. In particular, the efficient collective density function is uniform when agents are logarithmic $(\gamma=1)$. We represented in Figure 5 the efficient collective beliefs either when $\gamma=10$, and when $\gamma=0.1$. When $\gamma=10$, the efficient density function is hump-shaped, whereas it is 


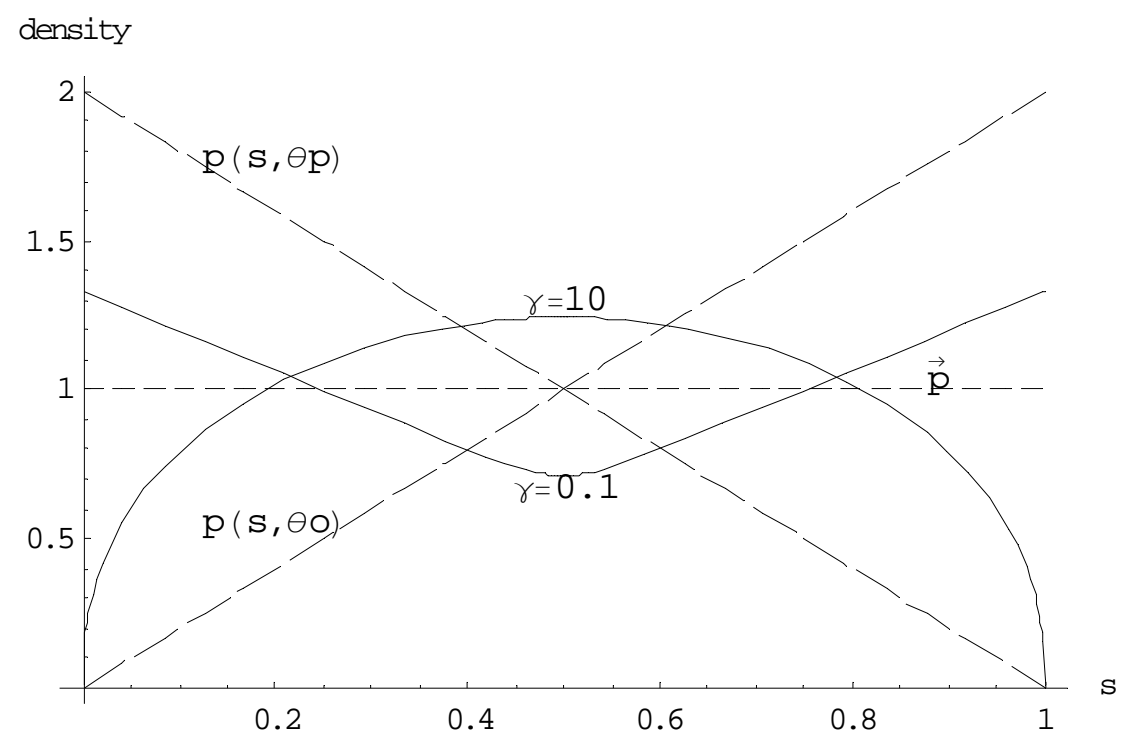

Figure 5: The collective density function for $u(c, \theta)=c^{1-\gamma} /(1-\gamma), p\left(s, \theta_{p}\right)=$ $2-2 s$, and $p\left(s, \theta_{o}\right)=2 s$.

U-shaped in the case of $\gamma=0.1$. This is a direct consequence of Corollary 2, since any move from $s=0.5$ towards the bounds of interval $[0,1]$ yields more disagreement.

\section{The equity premium and doubts on the oc- currence of a boom}

What are the implications of these results on the equity premium? The equity premium is the expected excess return that is obtained at equilibrium when accepting to bear the collective risk rather than investing in a risk free bond. There is a specific difficulty to use this concept when there is no agreed-upon probability distribution for the excess return. We consider the position of the econometrician who can use a long time series of observations. The sample average state probability $\vec{p}(s)$ equals the objective probability of state $s$. For some reasons, investors disagree on these state probabilities, but we assume that their expectations are unbiased on average: $E p(s, \vartheta)=\vec{p}(s)$ 
for all $s \in S .^{10}$

The group is reinterpreted as an exchange economy à la Lucas (1978), which implies that the equilibrium condition is $\omega(s)=z(s)$ for all $s$, where $\omega(s)$ and $z(s)$ are respectively the mean endowment and the mean consumption in state $s$. The first-order condition of program (20) can be rewritten as an equilibrium condition as follows:

$$
v_{z}(\omega(s), s)=\pi(s)
$$

where $\pi(s)$ is the price of the Arrow-Debreu security associated to state $s$. Suppose that investors have state-independent ISHARA utility functions. We know from Proposition 3 that $v_{z}$ is multiplicatively separable in that case: $v_{z}(\omega, s)=p^{v}(s) \nu^{\prime}(\omega)$. The risk free rate equals

$$
R^{f}={ }_{S}^{\cdot \mathbf{Z}} \pi(s) d s^{\nu^{-1}}={ }_{S}^{\cdot \mathbf{Z}} p^{v}(s) \nu^{\prime}(\omega(s)) d s^{{ }^{-1}}
$$

The price of equity equals

$$
={ }_{S}^{\mathbf{Z}} \omega(s) \pi(s) d s={ }_{S}^{\mathbf{Z}} p^{v}(s) \omega(s) \nu^{\prime}(\omega(s)) d s .
$$

The objective expected payoff of equity is equal to $\vec{\mu}={ }_{S}^{\mathrm{R}} \vec{p}(s) \omega(s) d s$. As in Abel (2002), the objective equity premium is thus equal to

$$
\phi=\frac{\vec{\mu}}{P^{e} R^{f}}=\frac{{ }_{S}^{\mathbb{R}} \vec{p}\left(s_{\mathbf{R}} \omega(s) d s_{S}^{\mathbf{\alpha} \boldsymbol{R}}{ }_{S}^{v}(s) \nu^{\prime}(\omega(s)) d s^{\mathbf{\alpha}}\right.}{{ }_{S} p^{v}(s) \omega(s) \nu^{\prime}(\omega(s)) d s} .
$$

When beliefs are homogeneous, $p^{v}($.$) and \vec{p}($.$) coincide, and the calibration$ of the parameters in equation (47) using historical data yields an equity premium that is much smaller than the average equity premium observed on financial markets during the last century. In this section, we examine whether the heterogeneity of beliefs could explain this puzzle.

It is intuitive that the equity premium would be increased if the representative agent would perceive equity as riskier than what is suggested by the econometrician. A larger equity premium would then be necessary to compensate for the larger subjective risk. Observe that the example summarized

\footnotetext{
${ }^{10}$ This is in sharp contrast with Abel (2002) who assumes alternatively that investors share their beliefs $p(s, \theta)=p(s) \forall \theta$, which are systematically biased with respect to the objective probability distribution $p^{*}$.
} 
in Figure 5. Suppose that aggregate wealth is equal to $s$ so that the horizontal axis in this figure measures wealth. The objective distribution of wealth is therefore uniform on $[0,1]$ in this example. When relative risk aversion is larger than unity, the divergence of opinions tends to reduce the probability of the extreme states, and to raise the probability of medium-wealth states. This means that the representative agent perceives the collective risk as smaller than what is given by the objective distribution. Following Proposition 7 , this is due to the fact that the divergence of opinions is strongest for the probability of the extreme events. Under the assumption that the derivative of absolute risk aversion is less than unity, this reduces the probability of these events, thereby reducing the risk perceived by the representative agent. Solving the equity premium puzzle would rather require concentrating the divergence of opinions to the medium-wealth states. This is not the most plausible assumption that one can do about the distribution of beliefs on financial markets. We will therefore not pursue this line of research. ${ }^{11}$

Let us alternatively assume that most of the divergence of opinions is concentrated on the probability of occurrence of a boom. The structure of individual beliefs are similar to those described in Figure 6. Suppose that $\omega(s)=s$, and assume that $S=] a, b]$. There is a boom if $s \in[b-\Delta, b]$. Individual $\theta$ believes that the probability of a boom state $s$ is $(1-\theta) \vec{p}(s)$. For all $s<b-\Delta-\varepsilon$, the subjective Rrobability of state $s$ for agent $\theta$ equals $(1-(1-\theta) k) \vec{p}(s) /(1-k)$, where $k={ }_{b-\Delta} \vec{p}(s) d s$ is the objective probability of a boom. In the small interval $I=[b-\Delta-\varepsilon, b-\Delta[$, the individual beliefs are selected in order to guarantee that $p(s, \theta)$ be differentiable with respect to $s$. By construction, we have that

$$
{ }^{s} \delta^{v}(s)=s \vec{\delta} p(s)
$$

for all $s$ except in interval $I$. We assume that $k$ and $\Delta$ are small, so that most of the disagreement is concentrated in the boom probability. It implies that the degree of disagreement is decreasing in interval $I$. Under the assumption that $T_{c}^{u} \leq 1$, Proposition 7 implies that ${ }^{s} \delta^{v}(s)$ must be smaller than ${ }^{s} \vec{\delta}^{p}(s)$. It implies that the representative agent has beliefs that are first-order stochastically dominated by the objective probability distribution. More specifically,

\footnotetext{
${ }^{11}$ An additional reason for this is that it is in general not true that an increase in the equity risk reduces the demand for equity by all risk-averse investors, thereby reducing the equilibrium price of equity. See Rothschild and Stiglitz (1971), Gollier (1995) and Abel (2002).
} 


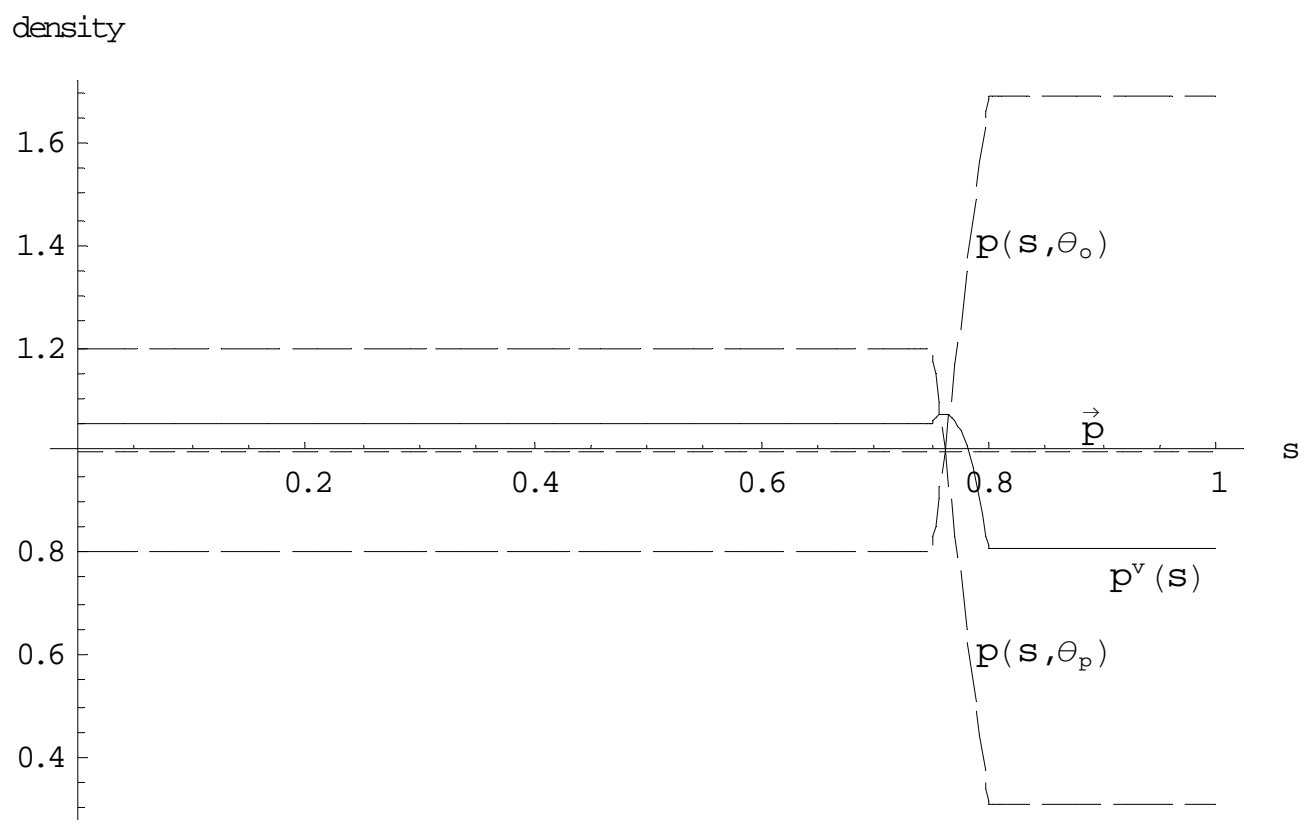

Figure 6: Most of the divergence of opinions is concentrated on the probability of occurence of a boom. Relative risk aversion $\gamma$ equals 10. The beliefs $p^{v}$ of the representative agent is first-order stochastically dominated by the mean beliefs $\vec{p}$.

the representative agent uses a density function $p^{v}$ such that

$$
p^{v}(s)=\begin{aligned}
& { }^{1 / 2} k_{1} \vec{p}(s) \geq \vec{p}(s) \quad \text { if } \quad s \leq b-\Delta-\varepsilon \\
& k_{2} \vec{p}(s) \leq \vec{p}(s) \text { if } \quad s \geq b-\Delta .
\end{aligned}
$$

with $k_{1}=\left(1-k_{2} k\right) /(1-k) \geq 1$. Perceiving equity returns as less favorable, the representative agent would reduce his demand for equity, thereby increasing the equilibrium equity premium.

Proposition 9 Suppose that most of the divergence in opinions is concentrated in the probability of occurrence of a boom. Suppose that agents have CRRA preferences $u(c)=c^{1-\gamma} /(1-\gamma)$ with $\gamma>1$. It implies that the heterogeneity of beliefs raises the equity premium.

Proof: It remains to prove that a transfer of probability mass from the wealthiest state to the other states raises the equity premium $\phi$. In Appendix 
B, we prove that $P^{e} R^{f}$ is reduced by a change in the probability density $p^{v}$ that transfers probability mass from any state with wealth per capita $\omega \geq \bar{\omega}$ uniformly the other states, as in (48). This critical wealth level $\bar{\omega}$ is strictly smaller than the upper bound of the support of the wealth distribution. It implies that the equity premium $\phi=\mu / P^{e} R^{f}$ is increased by the heterogeneity of beliefs when the divergence of opinions is concentrated in the probability of occurrence of the wealthiest state. $¥$

In the rest of this section, we consider a credible calibration exercise whose objective is to show that the effect of divergent opinions may have a strong impact on the equity premium. We assume that all agents have the same utility function with a constant relative risk aversion $\gamma$ equaling 4. Suppose that agents form expectations for the future growth rate of the economy from observing the realized growth rates of real GDP per capita in the U.S.A. over the 70's and 80's (source Penn-World data). The mean growth rate has been $1.72 \%$ per year, and the standard deviation equalled $2.48 \%$. This is not far from the historical mean (1.8\%) and standard deviation (3.56\%) that have been reported by Kocherlakota (1996) for the period 1889-1978. In a situation where agents agree on the probability distribution that would attach a probability $1 / 20$ to each of the 20 observations, the equilibrium equity premium would equal $0.25 \%$ per year, far below the average equity premium of $6 \%$ observed during the last century.

Suppose alternatively that agents disagree on the probability distribution of the growth rate of the economy. We gathered the five best years of these two decades, for which the growth rate exceeded 3.7\%. These states are referred to as boom states, whereas the other states are referred to as "normal" states . From our data, the objective probability of a boom equals $1 / 4$. The objective probability of the occurrence of a normal state is $3 / 4$. But, for some reason, agent of type $\theta$ attaches a probability $\theta$ not necessarily equal to $3 / 4$ to the occurrence of a normal state. The probability of occurrence of a boom is the only source of divergence of opinions. This means in particular that all agents agree on the probability of $1 / 5(1 / 15)$ of each of the observed growth rates conditional to a (no-)boom. We stress the fact that our simulations preserve the mean expectations which are equal to the objective ones. More precisely, we assume that the mean $\theta$ is $3 / 4$. There are two groups of equal size and with the same Pareto-weights. The members of the optimistic group believe that the probability of occurrence of a normal state is $\theta_{m}$ that is smaller than $3 / 4$, whereas the members of the pessimistic group believes that the probability of occurrence of a normal state is $1.5-\theta_{m}>3 / 4$. Con- 


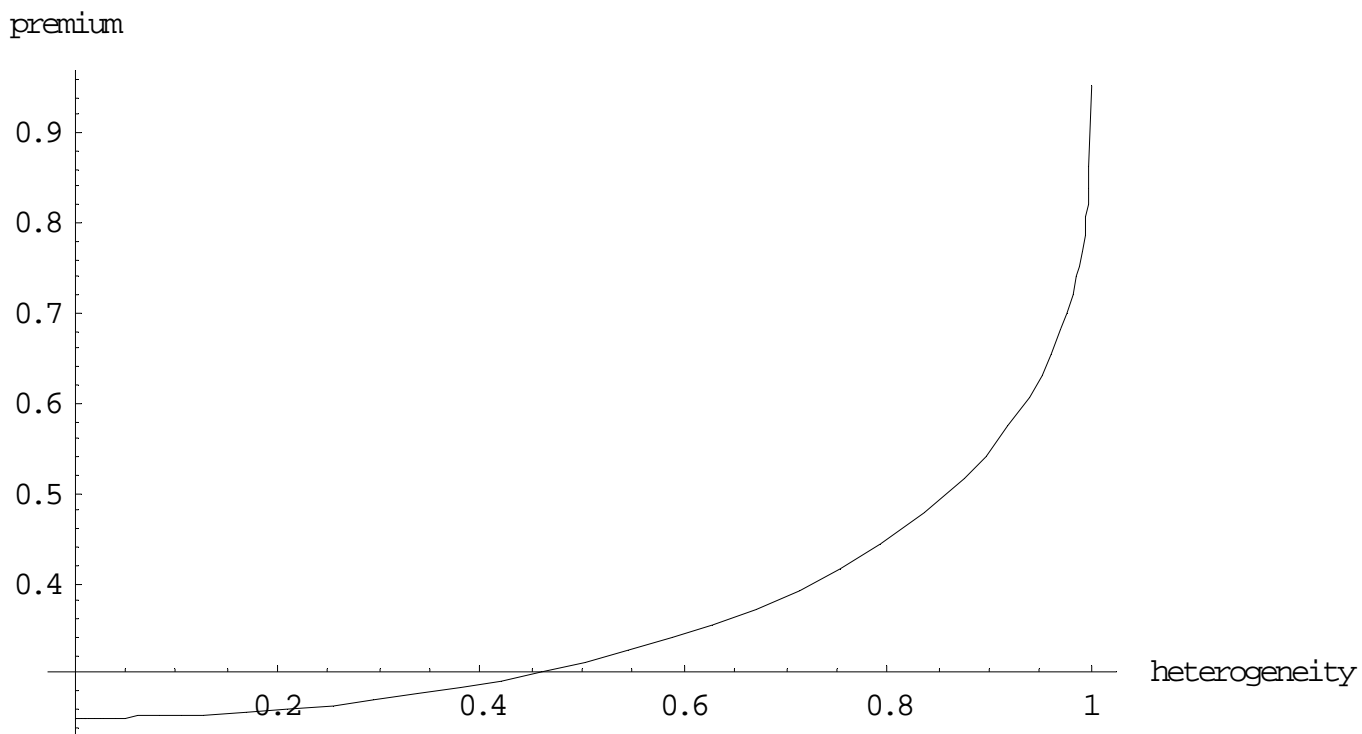

Figure 7: The equity premium in percent per year as a function of the degree of heterogeneity. The degree of heterogeneity is unity when $\theta$ is distributed as $(0.5,1 / 2 ; 1,1 / 2)$. It is zero for $\theta \sim(0,75,1 / 2 ; 0.75,1 / 2)$. More generally, the degree of heterogeneity is measured by $3-4 \theta_{m}$.

sider in particular case $\theta_{m}=1 / 2$, in which case the optimistic group believes that a boom will occur with certainty, whereas the pessimistic group believes that this event will occur only with probability $1 / 2$. With such a strong divergence of opinions on the occurrence of a boom, the equilibrium equity premium goes up to $0.96 \%$. It is far to explain the equity premium puzzle, but it multiplies almost by a factor 4 the equity premium that would have been observed without heterogeneous beliefs. In Figure 7, we describe the equity premium as a function of the degree of divergence of opinions. We see that the equity premium increases with the degree of heterogeneity at an increasing rate. The details of our computations are presented at the end of Appendix C.

We could have alternatively assumed that most of the divergence of opin- 
ions is concentrated on the probability of occurrence of a krach. Under the assumption that the derivative of absolute tolerance is less than unity, this would tend to reduce the equity premium. Why shouldn't we take into account of the extremely pessimistic agents who believe that the likelihood of a krach is high? Just because these agents will purchase insurance against the occurrence of the krach state. At the limit, they will stay out of the equity market. Only the optimistic agents will participate. That will have a negative impact on the equity premium.

\section{Conclusion}

Our aim in this paper was to characterize the probability distribution that should be used for collective decision making when individuals differ about their expectations. To examine this question, we assumed that agents can share risk efficiently, thereby relying on techniques borrowed from the theory of finance. The basic ingredient behind our results is that, in aggregating individual beliefs, one should favor the beliefs of agents that bear a larger share of the risk. However, the allocation of risk in the economy is endogenous and it depends upon the individual beliefs. Therefore, efficient risk allocations are more difficult to characterize under expectations disagreement. For example, it is not necessarily efficient to wash out diversifiable risks in that case. It may be efficient for agents to gamble against each others in spite of their risk aversion. Horsetrack betting is Pareto-improving when agents have different beliefs about the chances of the competing horses.

In an Arrow-Debreu framework, the risk exposure of an individual is a local concept that is measured by local differences in state consumption levels across states. As is well-known, the socially efficient local risk exposure for an agent is proportional to his local degree of absolute risk tolerance which measures the rate at which marginal utility decreases with consumption. We showed that this result remains true with heterogeneous beliefs. Associated to this concept of local risk tolerance, we developed a concept of local pessimism, which is measured by the rate at which the subjective state probability decreases across states. We showed that, locally, the degree of pessimism of the representative agent is the mean of individual degrees of pessimism weighted by the individual degrees of absolute risk tolerances. This result gives some flesh to the intuition that the collective beliefs should be biased in favor of the beliefs of the risk-takers. This result has several 
important consequences.

First, it implies that the socially efficient collective probability distribution depends upon the aggregate wealth level of the group. This is because the aggregate wealth level affects the way risks should be allocated in the group. However, when agents have the same HARA utility function, changes in aggregate wealth has no effect on the allocation of risks. This implies that the collective probability distribution is independent of wealth in that case. We showed that the identically-sloped HARA case is the only case in which such separability property between beliefs and utility holds. In all other cases, it is not possible to separate the contribution function $v(z, s)$ into the product of a state probability and a utility function, the first being independent of the consumption level and the second being independent of the state. In short, it implies that the representative agent has a state-dependent utility function.

Second, we derived various results that are useful to understand the effect of the divergence of opinions on the shape of the collective probability distribution. To do this, we defined the concept of increasing disagreement, and we assumed that agents have the same state-independent utility function. . In short, there is more disagreement about the probability of state $s^{\prime}$ than about the probability of state $s$ if the individual subjective probabilities are more dispersed in state $s^{\prime}$ than in state $s$. If the average degree of pessimism is the same in the two states, we showed that the collective degree of pessimism should be smaller in state $s^{\prime}$ with higher disagreement if and only if absolute risk aversion is decreasing. If we alternatively assume that the (Pareto-weighted) average subjective probability is the same in the two states, the collective degree of pessimism is positive if and only if the derivative of absolute risk tolerance is smaller than unity, a plausible hypothesis on preferences. It must be stressed that these results are purely local. They do not provide a global view about how the beliefs of the representative agent are affected by the heterogeneity of beliefs.

The last step is to link the structure of disagreement at the global level to the global properties of the collective probability distribution. When most disagreements are concentrated in the wealthier states, the collective distribution function is dominated by the average individual probability distribution in the sense of first-order stochastic dominance. This tends to raise the equity premium. We showed in a simple numerical example that the heterogeneity of individual beliefs may have a sizeable effect on the equity premium.

The critical assumption of this model is that the group can allocate risk 
efficiently. This assumption is difficult to test. For example, the efficient coverage of earthquake coverage in various regions can be interpreted in two ways. The optimistic view is that homeowners are less pessimistic than insurers about the risk, which implies that the low insurance coverage is socially efficient. But alternatively, it could be interpreted as a proof that markets are incomplete. A similar problem arises to explain the insurance crisis after $9 / 11 / 01$, or about the difficulty to share the risk related to global warming on an international basis. A possible extension of this work would be to consider an economy with incomplete markets. 


\section{References}

Athey, S., (2001), Comparative statics under uncertainty: single crossing properties and log-supermodularity, Discussion paper, MIT.

Abel, A.B., (2002), An exploration of the effects of pessimism and doubt on asset returns, Journal of Economic Dynamics and Control, 26, 1075-1092.

Borch, K. (1960). "The Safety Loading of Reinsurance Premiums", Skandinavisk Aktuarietskrift 153-184.

Calvet, L., J.-M. Grandmont and I. Lemaire, (2001), Aggregation of heterogenous beliefs and asset pricing in complete financial markets, mimeo, Harvard University and CREST (Paris).

Cecchetti, S.G., P.-S. Lam and N.C. Mark, (2000), Asset pricing with distorted beliefs: Are equity returns too good to be true?, American Economic Review, 90, 787-805.

Constantinides, G.M., (1982), Intertemporal asset pricing with heterogeneous consumers and without demand aggregation, Journal of Business, 55, 253-67.

Drèze, J.H., (2001), Loss reduction and implicit deductibles in medical insurance, CORE discussion paper, U. of Louvain.

Drèze, J.H., and A. Rustichini, (2001), State-dependent utility and decision theory, in S. Barbera, P. Hammond, and C. Seidl, Eds, Handbook of Utility Theory, 2, Kluwer, Dordrecht.

Gollier, C., (1995), The Comparative Statics of Changes in Risk Revisited, Journal of Economic Theory , 66, 522-536.

Gollier, C., (2001), The economics of risk and time, MIT Press, Cambridge, Ma.

Gollier, C., (2002), Optimal dynamic portfolio risk with firstorder and second-order predictability, mimeo, University of Toulouse.

Gollier, C., and M.S. Kimball, (1996), Toward a systematic approach to the economic effects of uncertainty: characterizing utility functions, Discussion paper, University of Michigan. 
Hara, C., and C. Kuzmics, (2001), Representative consumer's risk aversion and efficient risk sharing rules, mimeo, Cambridge University.

Ingersoll, J., (1987), Theory of Financial decision making, Rowman and Littlefield, Totowa, New Jersey.

Kimball, M.S., (1990), Precautionary savings in the small and in the large, Econometrica, 58, 53-73.

Leland, H.E., (1980), Who Should Buy Portfolio Insurance?, Journal of Finance, 35, 581-596.

Nau, R.F., (2002), A generalization of Pratt-Arrow measure to non-expected-utility preferences and inseparable probability and utility, Management Science, forthcoming.

Rothschild, M. and J. Stiglitz, (1971), Increasing risk: II Its economic consequences, Journal of Economic Theory 3, 66-84.

Varian, H., (1985), Divergence of opinion in complete markets, Journal of Finance, 40, 309-317.

Wilson, R. (1968). "The theory of syndicates", Econometrica 36, 113-132. 


\section{Appendix A: The case of ISHARA preferences}

We have shown in section 2 that the concept of pessimism is more useful than probabilities themselves to examine the optimal risk exposure when markets are complete. The individual degree of pessimism is an index measuring probability differences across states. However, it may be useful to examine the direct effect of divergent beliefs on the efficient probability distribution. This can be done by first deriving the collective degree of pessimism $\delta^{v}$ from the above analysis and by then solving the differential equation

$$
p_{s}^{v}(z, s)=-{ }^{s} \delta^{v}(z, s) p^{v}(z, s) .
$$

Finally, the normalizing constant of this PDE must be selected in order to guarantee that ${ }_{S} p^{v}(z(s), s) d s=1$.

In this appendix, we examine the special case of ISHARA preferences (32) for which we know from proposition 3 that the differential equation is simplified by the fact that ${ }^{s} \delta^{v}$ is independent of $z$. It implies that the collective probability distribution $p^{v}$ is also wealth independent. Moreover, ISHARA preferences (32) yield an analytical solution for the aggregation problem. Indeed, in this particular case, the first-order condition to statedependent the Pareto program (19) implies that

$$
c(z, s, \theta)-a(\theta)=k(s)[\lambda(\theta) p(s, \theta)]^{1 / \gamma} .
$$

Since $T^{u}(c, \theta)=(c-a(\theta)) / \gamma$, property $(27)$ can be rewritten in the ISHARA case as

$$
s \delta^{v}(z, s)=\frac{E^{s} \delta^{p}(s, \vartheta) \lambda(\vartheta) p(s, \vartheta)^{\mathbf{i}_{1 / \gamma^{\nu}}}}{E^{\mathbf{h}} \lambda(\boldsymbol{\vartheta}) p(s, \boldsymbol{\vartheta})^{\mathbf{i}^{1 / \gamma}}},
$$

or equivalently, using the definition of pessimism,

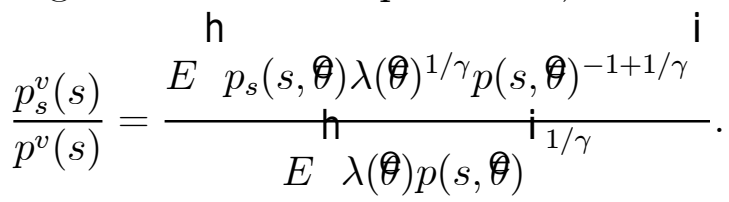

Obviously, the general solution to this differential equation has the following form:

$$
p^{v}(s)=C E^{\cdot}{ }^{\mathrm{h}} \lambda(\boldsymbol{\theta}) p(s, \boldsymbol{\theta})^{\mathbf{i}_{1 / \gamma^{\lrcorner \gamma}}},
$$


where $C$ is a constant. In order for $p^{v}$ be a probability density, we need to select the particular solution with

$$
\begin{aligned}
& { }_{E_{\boldsymbol{\theta}}}^{\mathrm{h}} \lambda(\boldsymbol{\vartheta}) p(s, \boldsymbol{\theta})^{\mathrm{i}_{1 / \gamma^{\circ} \gamma}} \\
& p^{v}(s)=\frac{{ }_{S} E_{\boldsymbol{\theta}} \lambda(\boldsymbol{\theta}) p(t, \boldsymbol{\theta})^{\mathrm{i}} \mathrm{i}_{1 / \gamma^{\lrcorner \gamma}} d t}{\mathrm{~h}} .
\end{aligned}
$$

Calvet, Grandmont and Lemaire (2001) obtained the same solution. Thus, in the ISHARA case, we can directly compute the socially efficient probability distribution of risk as a function of individual beliefs $p$, the Pareto weights $\lambda$, and the concavity coefficient $\gamma$. Two special cases are worthy to examine. Consider first the case with $\gamma$ tending to zero. This corresponds to risk-neutral preferences above a minimum level of subsistence. Under this specification, condition (51) is rewritten as

$$
p^{v}(s)=p^{0}(s)={ }_{d e f} \frac{\operatorname{Rax}_{\theta \in \Theta} \lambda(\theta) p(s, \theta)}{\max _{\theta \in \Theta} \lambda(\theta) p(t, \theta) d t} \text { for all } s . \text { (risk-neutral case) }
$$

With risk-neutral preferences, the efficient allocation produces a flip-flop strategy where the cake in state $s$ is entirely consumed by the agent with the largest Pareto-weighted probability associated to that state. It implies that the group will use a state probability $p^{0}$ proportional to it to determine its attitude toward risk ex ante.

In the case of logarithmic preferences $(\gamma=1)$, the denominator in (51) equals $E \lambda(\theta)$ since

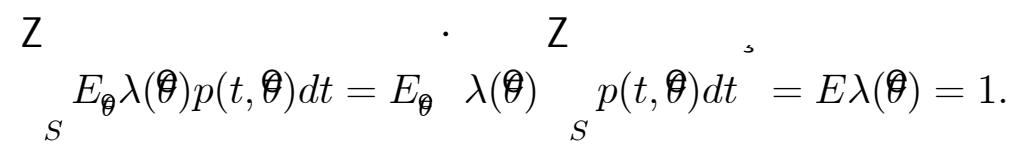

It implies that

$$
p^{v}(s)=p^{1}(s)=_{\text {def }} E \lambda(\boldsymbol{\Theta}) p(s, \boldsymbol{\vartheta}) \text { for all } s . \text { (logarithmic case) }
$$

With these Bernoullian preferences, the efficient probability that should be associated to any state $s$ is just the weighted mean $p^{1}(s)$ of the individual subjective probabilities of that state $s$. This is the limit case $T_{c}^{u} \equiv 1$ of the result presented in Proposition 7. 


\section{Appendix B: Change in beliefs and the equity premium}

In this Appendix, we show that a uniform transfer of probability mass from a high wealth state reduces the future price of equity $P^{e} R^{f}$. It implies that the equity premium $\phi=\mu / P^{e} R^{f}$ is reduced.

Proposition 10 Consider a random variable $\boldsymbol{9}(p)$ which is distributed as $\left(\boldsymbol{e}, p ; x_{0}, 1-p\right)$, with $x_{0} \in R$ and $p \in[0,1]$. Suppose that the real-valued function $v$ is increasing and concave. The future price of equity

$$
F P^{e}(p)=\frac{E \varphi(p) v^{\prime}(\varphi(p))}{E v^{\prime}(\varphi(p))}
$$

is decreasing in $p$ if and only if $x_{0}$ is larger than $\bar{x}=E \mathbf{e} v^{\prime}(\mathbb{e}) / E v^{\prime}(\mathbb{e})$, which is the risk-neutral mean of $\mathbb{e}$.

Proof: $F P^{e}(p)$ is decreasing in $p$ if and only if

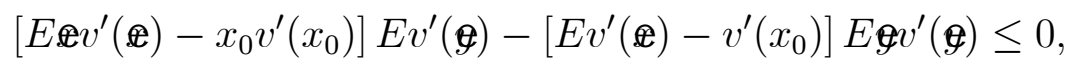

where $\boldsymbol{Q}$ is distributed as $\left(\mathbb{e}, p ; x_{0}, 1-p\right)$. This condition can be rewritten as

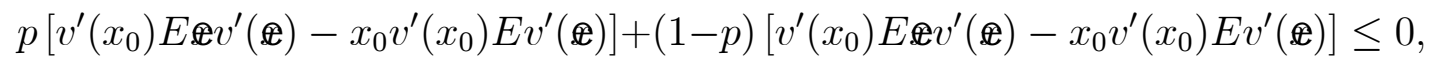
or, equivalently, as

$$
v^{\prime}\left(x_{0}\right)\left[E \circledast v^{\prime}(๕)-x_{0} E v^{\prime}(๕)\right] \leq 0 .
$$

This is true if and only if $x_{0}$ is larger than $\bar{x}=E £ v^{\prime}(\mathbb{e}) / E v^{\prime}(\mathbb{e})$. $¥$

\section{Appendix C: Numerical illustration}

In this appendix, we provide the details of the numerical illustration presented at the end of section 8. We assume that all agents have the same state-independent utility function $u(c)=-c^{-3} / 3$, which yields a constant relative risk aversion equaling $\gamma=4$. We consider the Pareto-efficient solution with equal weights. The percentage yearly growth rate of real GDP per capita for period 1970-1990 in the United States is

$$
\begin{aligned}
D \log G D P= & (2.04,3.74,4.4,-2.14,-2.89,4.13,3.78,3.94,1.26,-0.02, \\
& 1.34,-4.06,2.57,5.94,1.90,1.65,1.96,2.96,2.13,-0.23)
\end{aligned}
$$


(source Penn-World data). Let $\boldsymbol{\Theta}_{1}$ be the uniformly distributed and discrete random variable whose support is given by the 15 smallest elements of $D \log G D P$. Similarly, let $\boldsymbol{\Theta}_{2}$ be the uniformly distributed and discrete random variable whose support is given by the 5 largest elements of $D \log G D P$ . The "objective" risk on the growth rate is the random variable which is distributed as $\left(\Theta_{1}, 3 / 4 ; \Theta_{2}, 1 / 4\right)$. Its mean equals $E \Theta=\vec{\mu}=1.0172$, which implies a mean growth rate of real GDP per capita of $1.72 \%$ per year during the period. When all agents have the same beliefs that is represented by $\Theta$, the equity premium that sustains the corresponding Pareto-efficient allocation equals

$$
\phi=\frac{E \otimes E u^{\prime}(\Theta)}{E \otimes u^{\prime}(\Theta)}=1.0025,
$$

or $0.25 \%$ per year.

We now consider an economy with heterogenous beliefs. Agent $\theta$ has beliefs on the growth rate per capita that are distributed as $(\theta) \sim\left(\boldsymbol{Y}_{1}, \theta ; \boldsymbol{q}_{2}, 1-\right.$ $\theta)$. Let types be distributed as $\theta \sim\left(\theta_{m}, 1 / 2 ; 1.5-\theta_{m}, 1 / 2\right)$, so that the mean type is $E \theta=3 / 4$. It means that individual expectations are equal to $\theta$ on average. It implies that $p(\omega, \theta)=\theta / 15$ if $\omega$ is in the support of $\boldsymbol{e}_{1}$, and $p(\omega, \theta)=(1-\theta) / 5$ if $\omega$ is in the support of $\boldsymbol{\Theta}_{2}$. From Appendix B, we know that the beliefs $p^{v}($.$) of the representative agent is such that$

$$
p^{v}(\omega)=\frac{\mathfrak{f}_{\left(\theta_{m} / 15\right)^{1 / \gamma}+\left(\left(1.5-\theta_{m}\right) / 15\right)^{1 / \gamma}}^{\mathbf{p}_{\gamma}}}{15\left[\left(\theta_{m} / 15\right)^{1 / \gamma}+\left(\left(1.5-\theta_{m}\right) / 15\right)^{1 / \gamma}\right]^{\gamma}+5\left[\left(\left(1-\theta_{m}\right) / 5\right)^{1 / \gamma}+\left(\left(\theta_{m}-0.5\right) / 5\right)^{1 / \gamma}\right]^{\gamma}}
$$

if $\omega$ is in the support of $\Theta_{1}$, and

$$
p^{v}(\omega)=\frac{f_{\left(\left(1-\theta_{m}\right) / 5\right)^{1 / \gamma}+\left(\left(\theta_{m}-0.5\right) / 5\right)^{1 / \gamma}}^{\text {pq }^{\text {q }}}}{15\left[\left(\theta_{m} / 15\right)^{1 / \gamma}+\left(\left(1.5-\theta_{m}\right) / 15\right)^{1 / \gamma}\right]^{\gamma}+5\left[\left(\left(1-\theta_{m}\right) / 5\right)^{1 / \gamma}+\left(\left(\theta_{m}-0.5\right) / 5\right)^{1 / \gamma}\right]^{\gamma}}
$$

if $\omega$ is in the support of $\boldsymbol{\Theta}_{2}$. It simplifies to

$$
p^{v}(\omega)=\begin{array}{ccc}
k_{1} / 15 & \text { if } \omega \text { is in the support of } \boldsymbol{e}_{1} ; \\
\left(1-k_{1}\right) / 5 & \text { if } \omega \text { is in the support of } \boldsymbol{\Theta}_{2} .
\end{array}
$$

with

$$
k_{1}=\frac{\mathfrak{f}_{\theta_{m}{ }^{1 / \gamma}+\left(1.5-\theta_{m}\right)^{1 / \gamma}}}{\left[\theta_{m}{ }^{1 / \gamma}+\left(1.5-\theta_{m}\right)^{1 / \gamma}\right]^{\gamma}+\left[\left(1-\theta_{m}\right)^{1 / \gamma}+\left(\theta_{m}-0.5\right)^{1 / \gamma}\right]^{\gamma}}
$$


It yields the following pricing formula:

$$
\phi=\frac{E \boldsymbol{e} \cdot{ }^{f} k_{1} E \boldsymbol{\Theta}_{1}^{-\gamma}+\left(1-k_{1}\right) E \boldsymbol{e}_{2}^{-\gamma} \not \alpha}{k_{1} E \boldsymbol{\Theta}_{1}^{1-\gamma}+\left(1-k_{1}\right) E \boldsymbol{\Theta}_{2}^{1-\gamma}}
$$

For example, when $\theta$ is distributed as $(0.5,1 / 2 ; 1,1 / 2)$, i.e., when $\theta_{m}=0.5$, we obtain that $k_{1} \simeq 0.96$. This is to be compared to $k_{1}=0.75$ in the homogenous case with $\theta_{m}=0.75$. This represents a massive transfer of probability mass to the lower wealth states compared to the objective distribution. Because

$$
E \Theta_{1}^{-\gamma}=0.973, \quad E \Theta_{2}^{-\gamma}=0.894, \quad E \Theta_{1}^{1-\gamma}=0.978, \quad E \Theta_{2}^{1-\gamma}=0.878,
$$

we conclude that $\phi=1.0096$, yielding an equity premium equaling $0.96 \%$. In Figure 7, we evaluate the equity premium for other values of $\theta_{m}$. 


\title{
CESifo Working Paper Series
}

\author{
(for full list see www.cesifo.de)
}

841 Robert Fenge and Martin Werding, Ageing and the Tax Implied in Public Pension Schemes: Simulations for Selected OECD Countries, January 2003

842 Robert Fenge and Martin Werding, Ageing and Fiscal Imbalances Across Generations: Concepts of Measurement, January 2003

843 Giovanni Andrea Cornia, The Impact of Liberalisation and Globalisation on Income Inequality in Developing and Transitional Economies, January 2003

844 Peter Fredriksson and Per Johansson, Program Evaluation and Random Program Starts, January 2003

845 Bernd Hayo and Matthias Wrede, Fiscal Equalisation: Principles and an Application to the European Union, January 2003

846 Syed M. Ahsan and Jaideep Oberoi, Inequality, Well-being and Institutions in Latin America and the Caribbean, January 2003

847 Chang Woon Nam and Doina Maria Radulescu, The Role of Tax Depreciation for Investment Decisions: A Comparison of European Transition Countries, January 2003

848 V. Bhaskar and Steinar Holden, Wage Differentiation via Subsidised General Training, January 2003

849 Paloma Lopez-Garcia, Labour Market Performance and Start-up Costs: OECD Evidence, January 2003

850 Christian Keuschnigg and Soren Bo Nielsen, Public Policy for Start-up Entrepreneurship with Venture Capital and Bank Finance, January 2003

851 Yin-Wong Cheung, Menzie D. Chinn, and Eiji Fujii, China, Hong Kong, and Taiwan: A Quantitative Assessment of Real and Financial Integration, January 2003

852 Gregory D. Hess, The Economic Welfare Cost of Conflict: An Empirical Assessment, February 2003

853 Douglas J. Cumming and Jeffrey G. MacIntosh, Comparative Venture Capital Governance. Private versus Labour Sponsored Venture Capital Funds, February 2003

854 Eckhard Janeba and John Douglas Wilson, Decentralization and International Tax Competition, February 2003

855 Tapio Palokangas, Capital Accumulation and Employment Cycles in a Model of Creative Destruction, February 2003 
856 Brendan Walsh, When Unemployment Disappears: Ireland in the 1990s, February 2003

857 Luis H. R. Alvarez and Erkki Koskela, A General Approach to the Stochastic Rotation Problem with Amenity Valuation, February 2003

858 Christian Schultz, Strategic Campaigns and Redistributive Politics, February 2003

859 Ernst Fehr and Joseph Henrich, Is Strong Reciprocity a Maladaptation? On the Evolutionary Foundations of Human Altruism, February 2003

860 Haizhou Huang, Dalia Marin, and Chenggang Xu, Financial Crisis, Economic Recovery and Banking Development in Former Soviet Union Economies, February 2003

861 Pedro Cardoso and Bernard M.S. van Praag, How Sustainable Are Old-age Pensions in a Shrinking Population with Endogenous Labour Supply?, February 2003

862 Volker Meier, Efficient Transfer of Aging Provisions in Private Health Insurance, February 2003

863 Edward Castronova, Theory of the Avatar, February 2003

864 Robert S. Chirinko, Hans van Ees, Harry Garretsen, and Elmer Sterken, Investor Protections and Concentrated Ownership: Assessing Corporate Control Mechanisms in the Netherlands, February 2003

865 Bernard M.S. van Praag and Pedro Cardoso, The Mix Between Pay-as-you-go and Funded Pensions and what Demography has to do with it, February 2003

866 Ernst Fehr, Urs Fischbacher, Bernhard von Rosenbladt, Jürgen Schupp, and Gert G. Wagner, A Nation-Wide Laboratory. Examining Trust and Trustworthiness by Integrating Behavioral Experiments into Representative Survey, February 2003

867 Frank Heinemann, The Inflationary Impact of Wage Indexation, February 2003

868 Eytan Sheshinski, Bounded Rationality and Socially Optimal Limits on Choice in a Self-Selection Model, February 2003

869 M. Hashem Pesaran, Estimation and Inference in Large Heterogenous Panels with Cross Section Dependence, February 2003

870 Luis H. R. Alvarez and Erkki Koskela, On the Tree-Cutting Problem under Interest Rate and Forest Value Uncertainty, February 2003

871 Norbert Berthold and Rainer Fehn, Unemployment in Germany: Reasons and Remedies, February 2003

872 Clemens Fuest, Bernd Huber, and Philipp Tilleßen, Tax Policy and Entrepreneurship in the Presence of Asymmetric Information in Capital Markets, February 2003

873 Eytan Sheshinski, Optimum and Risk-Class Pricing of Annuities, February 2003 
874 Willi Leibfritz, Paul O'Brien and Jean-Christophe Dumont, Effects of Immigration on Labour Markets and Government Budgets - An Overview, February 2003

875 M. Hashem Pesaran and Allan Timmermann, How Costly is it to Ignore Breaks when Forecasting the Direction of a Time Series?, February 2003

876 Thorvaldur Gylfason and Gylfi Zoega, Education, Social Equality and Economic Growth: A View of the Landscape, February 2003

877 Robin Boadway and Jean-François Tremblay, Public Economics and Startup Entrepreneurs, February 2003

878 Erkki Koskela and Roope Uusitalo, The Un-Intended Convergence: How the Finnish Unemployment Reached the European Level, February 2003

879 Robert Fenge and Volker Meier, Pensions and Fertility Incentives, February 2003

880 Eytan Sheshinski, Note on Income Taxation and Occupational Choice, February 2003

881 A B Atkinson, Income Inequality in OECD Countries: Data and Explanations, February 2003

882 Thomas Gehrig and Rune Stenbacka, Venture Cycles: Theory and Evidence, February 2003

883 Ralf Becker and Thomas Hellmann, The Genesis of Venture Capital - Lessons from the German Experience, March 2003

884 Eytan Sheshinski, Note on the Optimum Pricing of Annuities, March 2003

885 Paul De Grauwe and Magdalena Polan, Globalisation and Social Spending, March 2003

886 F. van der Ploeg, Do Social Policies Harm Employment and Growth?, March 2003

887 Mirjam van Praag, Initial Capital Constraints Hinder Entrepreneurial Venture Performance: An empirical analysis, March 2003

888 Bernard Steunenberg, Coordinating Sectoral Policymaking: Searching for Countervailing Mechanisms in the EU Legislative Process, March 2003

889 Eytan Sheshinski, Optimum Delayed Retirement Credit, March 2003

890 Frederick van der Ploeg, Rolling Back the Public Sector - Differential effects on employment, investment and growth, March 2003

891 Paul De Grauwe and Marc-Alexandre Sénégas, Monetary Policy in EMU when the Transmission is Asymmetric and Uncertain, March 2003

892 Steffen Huck and Kai A. Konrad, Strategic Trade Policy and the Home Bias in Firm Ownership Structure, March 2003 
893 Harry Flam, Turkey and the EU: Politics and Economics of Accession, March 2003

894 Mathias Hoffmann and Ronald MacDonald, A Re-examination of the Link between Real Exchange Rates and Real Interest Rate Differentials, March 2003

895 Badi H. Baltagi, Espen Bratberg, and Tor Helge Holmås, A Panel Data Study of Physicians' Labor Supply: The Case of Norway, March 2003

896 Dennis C. Mueller, Rights and Citizenship in the European Union, March 2003

897 Jeremy Edwards, Gains from Trade in Tax Revenue and the Efficiency Case for Trade Taxes, March 2003

898 Rainer Fehn and Thomas Fuchs, Capital Market Institutions and Venture Capital: Do They Affect Unemployment and Labour Demand?, March 2003

899 Ronald MacDonald and Cezary Wójcik, Catching Up: The Role of Demand, Supply and Regulated Price Effects on the Real Exchange Rates of Four Accession Countries, March 2003

900 R. Selten, M. Schreckenberg, T. Pitz, T. Chmura, and S. Kube, Experiments and Simulations on Day-to-Day Route Choice-Behaviour, April 2003

901 Stergios Skaperdas, Restraining the Genuine Homo Economicus: Why the Economy Cannot be Divorced from its Governance, April 2003

902 Yin-Wong Cheung, Menzie D. Chinn, and Antonio Garcia Pascual, What Do We Know about Recent Exchange Rate Models? In-Sample Fit and Out-of-Sample Performance Evaluated, April 2003

903 Mika Widgrén, Enlargements and the Principles of Designing EU - Decision-Making Procedures, April 2003

904 Phornchanok Cumperayot, Dusting off the Perception of Risk and Returns in FOREX Markets, April 2003

905 Kai A Konrad, Inverse Campaigning, April 2003

906 Lars P. Feld and Stefan Voigt, Economic Growth and Judicial Independence: Cross Country Evidence Using a New Set of Indicators, April 2003

907 Giuseppe Bertola and Pietro Garibaldi, The Structure and History of Italian Unemployment, April 2003

908 Robert A.J. Dur and Otto H. Swank, Producing and Manipulating Information, April 2003

909 Christian Gollier, Collective Risk-Taking Decisions with Heterogeneous Beliefs, April 2003 